\title{
Kelvin-Helmholtz disruptions in extended magnetized jet flows
}

\author{
H. Baty ${ }^{1}$ and R. Keppens ${ }^{2, \star}$
}

1 Observatoire Astronomique, 11 rue de l'Université, 67000 Strasbourg, France

e-mail: baty@astro.u-strasbg.fr

2 FOM-Institute for Plasma Physics Rijnhuizen, PO Box 1207, 3430 BE Nieuwegein, The Netherlands e-mail: keppens@rijnh.nl

Received 3 August 2005 / Accepted 5 October 2005

\section{ABSTRACT}

We numerically investigate the long-term temporal evolution of magnetized jets where the computational domain covers multiple wavelengths (up to 10) of the fastest growing Kelvin-Helmholtz unstable mode. The dynamical importance of the magnetic field, which is initially uniform and flow-aligned, varies over a significant range: the plasma $\beta$ in the jets ranges from $O(1000)$ (essentially hydrodynamical) down to $O(1)$ (equipartition jets). Our calculations of two-dimensional, longitudinally periodic, extended slab configurations identify an inverse cascade process in the overall disruption to a broadened and heated jet flow. This process occurs for transonic and supersonic flows as well, with rapid shock-dominated transients appearing in supersonic cases, and with characteristic differences depending on the initial jet width. For configurations with a jet velocity profile having a radius that is much larger than the vorticity thickness of the flow, the cascade proceeds early through pairing/merging of individual mode structures on both jet boundaries. Jets with radii of the order of the vorticity thickness are strongly unstable to sinuous deformations with boundary layer-layer interactions between vortex (transonic, weak magnetic field) and shock (supersonic, strong field) structures in a few sound crossing times. We back up these findings for planar jets with selected three-dimensional simulations of extended cylindrical jet configurations. These tend to have more small-scale fluctuations in their relaxed endstates. The timescales and overall scenario for the helical disruptions agree well with the 2D studies. This allows us to discuss the possible implications of our results in the context of magnetohydrodynamic stability of astrophysical jets.

Key words. instabilities - magnetohydrodynamics (MHD) - ISM: jets and outflows - methods: numerical - plasmas

\section{Motivation}

A fundamental question raised by the observations of astrophysical jets concerns the flow survival to internal instabilities. Indeed, many collimated flows are observed to propagate over very long distances with respect to their radial distances without being disrupted. This is for example the case of jets emanating from young stellar objects (YSO) and active galactic nuclei (AGN). Moreover, these supersonic jets are typically observed to terminate in a strong final shock when they encounter a denser external medium, and not as a consequence of the development of internal instabilities. On the other hand, such sheared flows are highly susceptible to drive Kelvin-Helmholtz (KH) instabilities (Birkinshaw 1991; Ferrari 1998). Indeed, high resolution hydrodynamic simulations of supersonic jets have clearly shown how the development of KH modes can nonlinearly lead to the formation of internal shocks, entrainment and mixing with the ambient material, and ultimately to disruption on a few sound transit time scales (Bodo et al. 1995, 1998). Thus, the corresponding time-scale of the disruption is

^ Astronomical Institute, Utrecht University, The Netherlands. too fast by more than one order of magnitude to account for the observations.

Among the possible mechanisms currently investigated to explain the latter apparent discrepancy, is the role of magnetic fields. Indeed, the presence of a large-scale magnetic field in accretion-disk-jet systems is now well established from observations (Ray et al. 1997; Pushkarev et al. 2005; Gabuzda et al. 2004) and jet launching models (e.g. Casse \& Keppens 2004, and references therein). The magnitude of the magnetic field is probably not sufficient to prevent or strongly weaken the (linear) growth of the KH instability (Baty 2005), except for particular magnetic field configurations (Appl \& Camenzind 1992). Thus, a crucial point concerns the role of a relatively weak magnetic field in the nonlinear stages of the KH instability. In a realistic jet, the magnetic field topology will be helical, as the toroidal field component plays a crucial role for the collimation of the ejected flow. However, considering uniformly magnetized jets with only a longitudinal field component, has the advantage of unambiguously separating the effect of the magnetic field on $\mathrm{KH}$ modes from effects due to interaction with other magnetohydrodynamic instabilities (see, e.g. Baty \& Keppens 2002). 
In the literature, one can easily find a relatively large amount of papers presenting numerical simulations of the $\mathrm{KH}$ instability in uniformly magnetized jets (see review by Hardee 2004, and references therein). However, most of these studies have focused on the consequences for the evolution of the global flow morphology by using a spatial approach (Hardee et al. 1992; Zhao et al. 1992). This is not the case of the numerical work done by Min $(1997 a, b)$ in twodimensional (2D) slab geometry, as the aim there was to study the long-term evolution of $\mathrm{KH}$ instabilities by using a temporal approach. Indeed, this latter work has highlighted the crucial role played by the antisymmetric $\mathrm{KH}$ mode in the flow disruption, in contrast to the negligible role of the symmetric mode. This is particularly true when the characteristic length scale of the shear flow layer is of order of the jet thickness. In the opposite limit where the shear layer is much smaller than the jet radius, the flow essentially survives and the final obtained state is a jet with a smoother velocity profile. Indeed, in this latter case, $\mathrm{KH}$ vortices developing at the jet/external medium interface lead to an enlarged transition profile with disruptive magnetic reconnection events, in a very similar way as obtained for a single shear flow layer (see e.g. Baty et al. 2003, also referenced as Paper I below). However, as a rather short periodic longitudinal length (equal approximately to the wavelength of the linearly dominant KH mode) has been adopted in Min's configurations, the possibility for large-scale coalescence was excluded. In Paper I, we demonstrated a strong coalescence process towards large scales occuring for a single magnetized shear flow layer, due to pairing/merging between adjacent $\mathrm{KH}$ vortices. The aim of the present work is to determine its implications for full 2D jet configurations, thereby extrapolating Min's studies of "individual" KH modes to configurations on large, longitudinally extended domains. We consider a wider range of sonic and Alfvénic Mach numbers, in order to qualify differences in the nonlinear evolutions for jets of widely different plasma beta. Finally, a few selected three-dimensional (3D) uniformly magnetized cases are investigated in order to adress the generality of our 2D results. It is often argued that, contrary to purely hydrodynamic models, the turbulence cascade properties are similar in 2D versus 3D MHD (Biskamp 1993). Significant differences in $3 \mathrm{D}$ could nevertheless be important, and are thus explored in this work.

The paper is organized as follows. The jet model and the numerical setup are presented in Sect. 2. In Sect. 3, we show the numerical results obtained for 2D slab configurations. Section 4 is devoted to the results concerning 3D cylindrical jets. We conclude with discussing the consequences of our findings in the context of astrophysical jets stability.

\section{The jet model and the numerical setup}

The full set of ideal compressible MHD equations is used in this work (see Paper I), implying that we consider "perfectly conducting" plasmas with very large kinetic and magnetic Reynolds numbers. This ideal description is a suitable proxy for many astrophysical environments including jets. However, small nonzero viscosity and resistivity are locally important as they allow for momentum exchange and magnetic reconnection, respectively. This is particularly the case during the later evolutionary stages of the MHD KH instability, when small scale structures are formed. We rely on the inherent numerical resistivity and viscosity in the employed conservative shock-capturing discretizations to mimic these dissipative processes. In this way, dissipation takes place through numerical truncation at the grid-cell level within the smallest resolved structures. The corresponding numerical dissipation coefficients are consequently not constant or uniform, but depend on the size of the structures. It is generally argued, on the basis of a number of results, that this approach provides an adequate model for subgrid-scale dissipation (see discussion in Jones et al. 1998). However, a convergence study by repeating the simulations using different grid resolutions is recommended.

Min (1997a,b) explicitly included a resistive term with dimensionless coefficient $\eta=0.01$ in the equations, clearly dominating the numerical resistivity due to discretization effects at the moderate spatial resolution of $100 \times 200$ used. For similar physical cases, we checked that our results already recover the conclusions published by Min for a resolution of $50 \times 150$ in correspondance with an estimated numerical Reynolds number $R_{\mathrm{m}} \equiv \epsilon /\left(L_{x} \eta\right)=O\left(10^{3}\right)$. In this definition of $R_{\mathrm{m}}$, we use a rescaling to the thickness $\epsilon$ of the shear flow layer instead of the simulation box length $L_{x}$, as the latter will be varied. The estimation is obtained by comparing resistive MHD runs of varying $\eta$ with the "ideal" approach. We may conclude that in using grid-adaptive high resolution simulations, we are able to reach magnetic Reynolds number up to a value of order $10^{5}$ (for the individual modes study).

\subsection{The jet model}

In this work, we consider a jet having an initial background flow directed along the longitudinal direction $(s)$ and sheared in the radial one $(r)$,

$V_{s}(r)=\frac{V}{2}\left[1-\tanh \left(\frac{F r}{R_{j}}-\frac{F R_{j}}{r}\right)\right]$,

with $V$ the amplitude of the jet velocity. The parameters $R_{j}$ and $F$ control the exact shape of the flow profile; $R_{j}$ is the jet radius (radial distance where $V_{s}=V / 2$ ), and $F=$ $R_{j} / \epsilon$ is a non-dimensional parameter controlling the characteristic thickness $\epsilon$ of the shear flow (i.e. of the vorticity). Typically, three flow profiles called $\operatorname{Pr} 1, \operatorname{Pr} 2$, and $\operatorname{Pr} 3$ are investigated in this work, in correspondance with $\left(R_{j}, F\right)$ equal to $(0.5,5),(0.25,2.5)$, and $(0.125,1.25)$ in our units respectively. In this way, we vary the radius $R_{j}$ while keeping $\epsilon$ constant and equal to 0.1 in our units. This choice of dimensionalization is important, as it ensures that we use the same spatial resolution through this initial (at $t=0$ ) vorticity thickness for all profiles. For the adaptive simulations, we enforce the highest effective resolution at $t=0$ throughout this thickness.

Note that the set of MHD equations is solved in Cartesian geometry, so the radial coordinate $r$ is $r \equiv|y|$ and $r \equiv\left(x^{2}+y^{2}\right)^{1 / 2}$ in $2 \mathrm{D}$ and $3 \mathrm{D}$, respectively. The longitudinal coordinate $s$ is $s \equiv x$ and $s \equiv z$ in $2 \mathrm{D}$ and $3 \mathrm{D}$, respectively. We assume that the jet is initially in pressure equilibrium with its surroundings. We take uniform temperature $P_{0}$ and density $\rho_{0}$, setting them 
Table 1. Initial parameters of individual modes simulations.

\begin{tabular}{cccccc}
\hline \hline Profile & $M_{\mathrm{s}}$ & $M_{\mathrm{A}}$ & $M_{\mathrm{f}}$ & $\beta$ & Resolution \\
\hline $\operatorname{Pr} 1-\operatorname{Pr} 2-\operatorname{Pr} 3$ & 1 & 7 & 0.99 & 59 & $50 \times 150,100 \times 300,150 \times 450,200 \times 600,250 \times 750,300 \times 900$ \\
$\operatorname{Pr} 1-\operatorname{Pr} 2-\operatorname{Pr} 3$ & 1.4 & 7 & 1.37 & 30 & $50 \times 150,100 \times 300,150 \times 450,200 \times 600,250 \times 750,300 \times 900$ \\
$\operatorname{Pr} 1-\operatorname{Pr} 2-\operatorname{Pr} 3$ & 1 & {$[2-500]$} & {$[0.89-1]$} & {$\left[4.8-3 \times 10^{5}\right]$} & $200 \times 600$ \\
$\operatorname{Pr} 1-\operatorname{Pr} 2-\operatorname{Pr} 3$ & 1.4 & {$[2-500]$} & {$[1.15-1.4]$} & {$\left[2.45-1.5 \times 10^{5}\right]$} & $200 \times 600$ \\
\hline
\end{tabular}

All simulations have been carried out in a 2D rectangular domain with $0 \leq x \leq L_{x}$ and $-L_{y} \leq y \leq L_{y}$, with $L_{x}=1$ and $L_{y}=1.5$. We use either a symmetric or antisymmetric perturbation. The transonic and supersonic cases are obtained for $V=1.29$, and $V=1.806$ respectively.

Table 2. Initial parameters of 2D extended simulation domains.

\begin{tabular}{cccccc}
\hline \hline Profile & $M_{\mathrm{s}}$ & $M_{\mathrm{A}}$ & $M_{\mathrm{f}}$ & $\beta$ & Resolution \\
\hline $\operatorname{Pr} 1-\operatorname{Pr} 3$ & 1 & 7 & 0.99 & 59 & $800 \times 800,1600 \times 1600$ \\
$\operatorname{Pr} 1-\operatorname{Pr} 3$ & 1.4 & 7 & 1.37 & 30 & $800 \times 800,1600 \times 1600$ \\
$\operatorname{Pr} 1-\operatorname{Pr} 3$ & 3 & 7 & 2.76 & 6.54 & $800 \times 800,1600 \times 1600$ \\
$\operatorname{Pr} 3$ & 1 & 50 & 1 & 3004 & $1600 \times 1600$ \\
\hline
\end{tabular}

All simulations have been carried out with $L_{x}=10, L_{y}=4$, and using an initially white noise perturbation. The transonic and two supersonic cases $\left(M_{\mathrm{s}}=1.4\right.$, and $\left.M_{\mathrm{s}}=3\right)$ are obtained for $V=1.29, V=1.806$, and $V=3.87$ respectively.

to unity and defining thus our normalization. The flow is embedded in an initially uniform longitudinal magnetic field of amplitude $B_{0}$, that must be weak enough in order to have a linearly unstable configuration. Consequently, the sonic speed $c_{\mathrm{s}}$ is uniform and $c_{\mathrm{s}}=\left(\gamma P_{0} / \rho_{0}\right)^{1 / 2}=1.29$ in our units for an adiabatic index $\gamma=5 / 3$. We also use the following definitions for the sonic and Alfvénic Mach numbers, $M_{\mathrm{s}}=V / c_{\mathrm{s}}$, and $M_{\mathrm{A}}=V / V_{\mathrm{a}}$, where $V_{\mathrm{a}}$ is the Alfvén velocity. The fast Mach number is also defined as $M_{\mathrm{f}}=V /\left(c_{\mathrm{s}}^{2}+V_{\mathrm{a}}^{2}\right)^{1 / 2}$. The parameter values used in the different runs (nonlinear simulations) are listed in Tables 1 and 2.

\subsection{Numerical setup}

The nonlinear simulation results are obtained using the general finite-volume based Versatile Advection Code, VAC ${ }^{1}$ (Tóth 1996) and its grid-adaptive variant AMRVAC (Keppens et al. 2003). For the present study, we use the full set of ideal compressible MHD equations. We select the explicit one-step total variation diminishing (TVD) scheme with minmod/Woodward limiting in 2D/3D (Collela \& Woodward 1984; Harten 1983). This is a second-order accurate shock-capturing method making use of a Roe-type approximate Riemann solver. For some selected cases, an adaptive version of VAC, AMRVAC, is used. This automated adaptive mesh refinement (AMR) strategy allows for a grid-level dependent choice of the spatial discretization. We follow Keppens et al. (2003) and use the computationally beneficial combination of a robust two-step Total Variation Diminishing Lax-Friedrichs method on all but the finest grid-level, together with the less diffusive TVD scheme on the highest one. Note that all discretization methods in VAC,

\footnotetext{
1 See http://www.phys.uu.nl/ ${ }^{\text {toth }}$
}

and all combinations of grid level dependent spatial discretization methods in AMRVAC, are fully conservative. AMRVAC results employed a base spatial grid which is refined by adding finer level grids where a higher resolution is needed. Finer level grids are adjusted, inserted or removed by periodically checking if the grid structure should be altered in response to the flow dynamics. We employed four grid levels with a refinement factor of 2 between two consecutive levels. Refining was done based on a Richardson-type extrapolation procedure, using a weighted average of density, $s$-momentum, and longitudinal $B_{\mathrm{s}}$ components. Inclusion of the $s$-momentum component is needed to guarantee that the shear layer is resolved at the highest effective resolution from $t=0$.

To study instabilities in flowing plasmas, especially for making comparisons with observational characteristics, it is common to perform a spatial analysis in which one considers the development of an initial perturbation imposed at a given location, typically the jet inlet (see, e.g. Hardee 2004, and references therein). However, this approach necessarily limits the study to modes occuring within the transit time through the domain. For the sake of studying the long time evolution, a temporal approach is preferable to the spatial one. We focus here on this long-term temporal evolution of instabilities, leaving additional convective effects beyond the scope of the present paper. We can then consider an idealized problem assuming periodicity along the longitudinal direction. This restricts the longitudinal wavelengths $\lambda$ to Fourier components that can fit in the numerical domain of length $L_{\mathrm{s}}$, i.e. $\lambda=L_{\mathrm{s}} / n \leq L_{\mathrm{s}}$ with $n$ a positive integer. Taking $L_{\mathrm{s}}$ equal to the wavelength of the linearly fastest growing instability $\lambda_{\mathrm{m}}$ allows the detailed study of this individual mode, but prohibits the formation of structures on a larger scale. Large-scale coalescence is conversely allowed by taking $L_{\mathrm{s}}$ greatly exceeding $\lambda_{\mathrm{m}}$. We will quantify linear growth rates and corresponding wavelengths for our planar transonic to supersonic jets and vary the longitudinal domain in the nonlinear simulations accordingly. We use free outflow boundaries on the lateral sides. These artificial lateral boundaries are taken sufficiently far enough away from the jet core to eliminate any effect on the long-term dynamics.

The unstable background jet configuration described above is perturbed by adding different forms of small amplitude transverse velocity, typically one percent of $V$, at the initial time $t=0$. For runs designed to study individual modes, a symmetric/antisymmetric perturbation is taken mainly located at the jet radius, in order to follow the varicose/sinuous mode. However, a white noise perturbation using a random number generator is 
more appropriate to let all unstable wavelengths grow in a natural way, in configurations having a long computational domain length that exceeds $\lambda_{\mathrm{m}}$. More explicitely, we use in the latter case (see also Paper I), $v_{r}=\delta V \exp \left[-\left(\frac{r-R_{j}}{2 \epsilon}\right)^{2}\right] \times \operatorname{ran}(s)$, where the function $\operatorname{ran}(s)$ represents a random number generator in the range $[-0.5: 0.5]$ and $\delta V=0.01$ quantifies the amplitude of the velocity perturbation.

\section{Results for two-dimensional jets}

The use of a two-dimensional slab configuration for representing the dynamics of jets allows us to simulate the evolution of instabilities with high resolution without prohibitive computational costs. There is a certain correspondence in the mode nomenclature between 2D and 3D configurations. For example, the varicose and sinuous instabilities that develop in a slab jet are analogous to the $m=0$ pinch and $m=1$ kink instabilities (respectively) of a cylindrical jet, $m$ being the azimuthal mode number. We start in Sect. 3.1 with quantifying the linear unstable modes for the different profiles and their nonlinear evolution on short domains. This provides the reference for the new extended domain studies in Sect. 3.2.

\subsection{Individual modes}

With the aid of the LEDAFLOW code Nijboer et al. (1997), we can compute all MHD waves and instabilities of a given one-dimensional stationary equilibrium. For the planar jet flow considered here, the equilibrium is quantified by the sonic and Alfvénic Mach numbers and the parameter $F=R_{j} / e$, and the linearised compressible MHD equations are then solved for perturbations with dependency $\exp \left(-\mathrm{i} \omega t+\mathrm{i} k_{x} x\right)$. Using a finite element discretization for the radial variation of the linear modes, we can compute eigenvalues and corresponding eigenfunctions under the assumption of a perfectly conducting, wall-bounded slab. These artificial lateral boundaries are always placed at $r= \pm 1.5$, so that narrower jets are effectively further separated from these walls. This allows us to approximate modes even with considerable eigenfunction variation in the external medium, although true leaky modes damped by radial acoustic power leakage are strictly speaking not well represented. We recall that the linear stability threshold (for developing $\mathrm{KH}$ modes) of transonic $M_{\mathrm{s}}=1$ shear flow layers is $M_{\mathrm{A}} \gtrsim 2$, it corresponds to weak enough initial magnetic fields (see Paper I and references therein). KH instabilities can be nonlinearly stabilized (i.e. after a small amount of quasi-linear growth) for the somewhat restricted range $2 \lesssim M_{\mathrm{A}} \lesssim 4$. We will therefore concentrate on the regime that is qualified as disruptive, obtained for $4 \lesssim M_{\mathrm{A}} \lesssim 30$. A last very weak field regime is obtained when $M_{\mathrm{A}} \gtrsim 30$, essentially hydrodynamic in the early stages of the KH instability.

Figure 1 shows the growth rate (the imaginary part of $\omega=$ $\left.\omega_{R}+i \omega_{I}\right)$ of the unstable modes versus horizontal wavenumber $k_{x}$ for the wide transonic jet $\left(M_{\mathrm{s}}=1, M_{\mathrm{A}}=7\right.$ and $\left.\operatorname{Pr} 1\right)$. Two prominent unstable modes exist over a significant range of horizontal wavelength $\lambda_{x}=2 \pi / k_{x}$, corresponding to a sinuous (kink) or varicose (sausage) perturbation of the slab jet. These

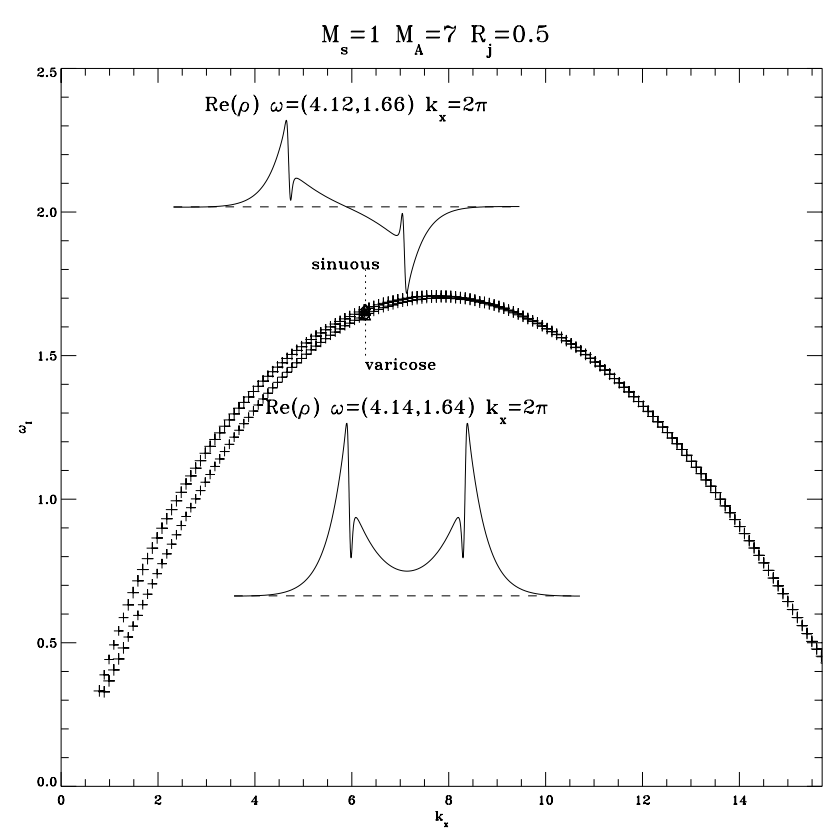

Fig. 1. Growth rate $\omega_{I}$ versus horizontal wavenumber $k_{x}$ for the unstable modes for a wide $\operatorname{Pr} 1$ transonic jet with $M_{\mathrm{A}}=7$.

modes coincide in growth rate as soon as their wavelength becomes shorter than the jet diameter $\lambda_{x} \lesssim 2 R_{j}$. Indeed, at $k_{x}=2 \pi$, the sinuous mode is found for $\omega=(4.12,1.66)$ and the varicose mode has eigenfrequency $\omega=(4.14,1.64)$. The real part of the density eigenfunction is indicated for both modes in the figure, showing their different symmetry properties and their surface mode character. Note that the phase velocity of both modes is to a good approximation given by the local velocity of the jet at $r=R_{j}: \omega_{R} \approx V_{x}\left(R_{j}\right) k_{x}=4.06$. For wavelengths longer than the jet diameter, the sinuous mode is the fastest growing instability. The maximal linear growth is observed for wavenumber $k_{x} \approx 7.5$ which is above, but close to $k_{x}=2 \pi$. Figure 2 shows the same information for unstable modes of a narrow transonic jet $\left(M_{\mathrm{S}}=1, M_{\mathrm{A}}=7\right.$ and $P r 3$, top panel) and a narrow supersonic jet $\left(M_{\mathrm{s}}=3, M_{\mathrm{A}}=7\right.$ and $\operatorname{Pr} 3$, bottom panel). Over the entire range of wavenumbers shown, the horizontal wavelength now exceeds the jet diameter. As a result, for these narrower jets, a clear dominance of the sinuous instability is found, with a significant increase in growth rate. A marked difference in phase velocity occurs as well: for $k_{x}=2 \pi$, the phase velocity of the sinuous mode is significantly below the advective speed at $r=R_{j}$, while it is above it for the varicose mode. The same is true for the supersonic narrow jet, where the oscillation frequency for the indicated $k_{x}=\pi$ modes would be $V_{x}\left(R_{j}\right) k_{x}=6.08$. The eigenmode structure for the supersonic jet clearly shows the significant density variation in the external medium, for both sinuous and varicose modes, so the modes loose their surface character in more supersonic jet regimes (see also Ferrari 1998; Birkinshaw 1991). Maximal sinuous growth rate is observed at a wavenumber $k_{x} \approx 8.5$ and $k_{x} \approx 4.5$ for transonic and supersonic narrow jets, respectively. For the supersonic flows, new branches (in addition to the surface modes) of "body" modes become unstable for the same domain wavelengths. These latter modes are typical of two-shear 

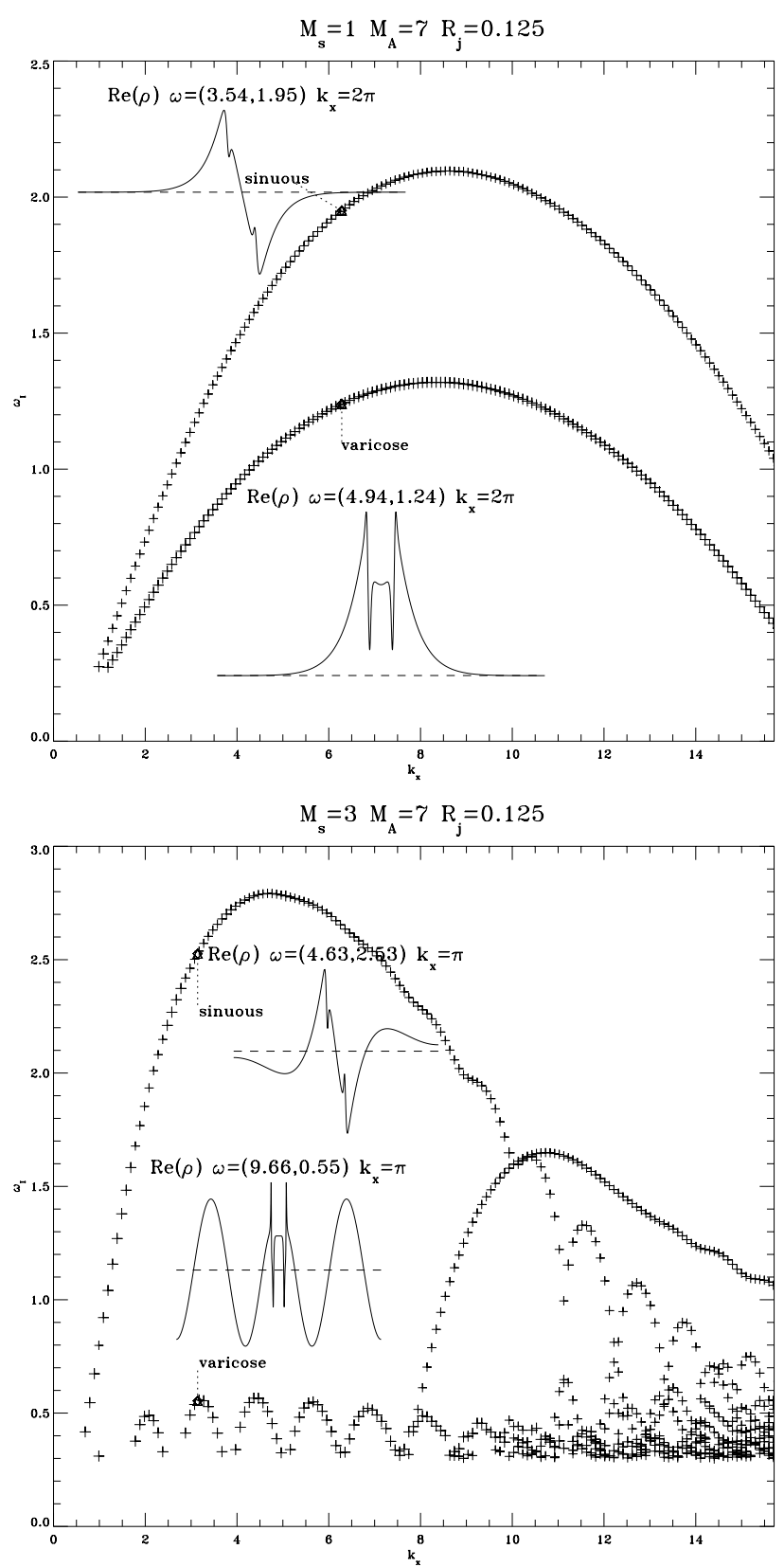

Fig. 2. Growth rate $\omega_{I}$ versus horizontal wavenumber $k_{x}$ for the unstable modes for narrow Pr 3 transonic (top) and supersonic (bottom) jets with $M_{\mathrm{A}}=7$.

layer configurations, as they become unstable by resonant reflections at the jet boundary.

With the above knowledge on the linear modes, we now first revisit the development of individual modes. This can be done by considering a periodic domain length $L_{x}$ approximately equal to the longitudinal wavelength of the linearly fastest growing mode. Min (1997a,b) performed such numerical simulations in resistive MHD, but focused on one case with sonic and Alfvénic Mach numbers $M_{\mathrm{s}}=1$ and $M_{\mathrm{A}}=10$.

Starting with the narrow $\operatorname{Pr} 3$ jet at sonic Mach number $M_{\mathrm{s}}=1$ and Alfvén Mach number $M_{\mathrm{A}}=7$, we run a static grid VAC simulation on a spatial domain where $L_{x}=1$, and $L_{y}=1.5$. The horizontal extent matches $k_{x}=2 \pi$, close to the

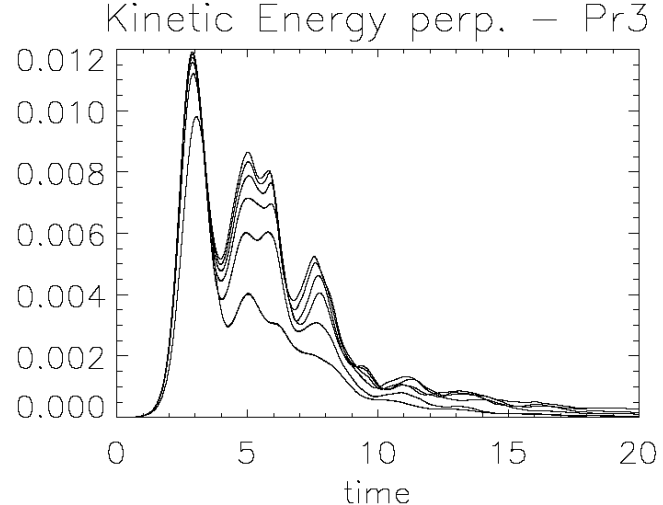

Fig. 3. Time evolution of the cross-stream kinetic energy $E_{k, y}$ (in arbitrary units) for the narrow $\operatorname{Pr} 3$ velocity profile perturbed with a sinuous mode. The different curves correspond to increasing spatial resolutions $50 \times 150,100 \times 300,150 \times 450,200 \times 600,250 \times 750$, and $300 \times 900$, from lower to higher amplitudes respectively. The physical case investigated is for $M_{\mathrm{s}}=1$, and $M_{\mathrm{A}}=7$.

maximally growing mode. We perturbed with either a sinuous or a varicose mode. The resolution taken is $200 \times 600$ grid points in the $x \times y$ domain, capturing the essential dynamics. For example, a convergence study showing the time evolution of the cross-stream kinetic energy $E_{k, y}$ averaged over the spatial domain volume $V_{b}=2 \times L_{x} \times L_{y}$,

$E_{k, y}=\frac{1}{V_{b}} \int_{V_{b}} \frac{\rho V_{y}^{2}}{2} \mathrm{~d} x \mathrm{~d} y$,

associated to a sinuous $\mathrm{KH}$ mode of a $\operatorname{Pr} 3$ velocity profile, is illustrated in Fig. 3 for 6 spatial resolutions ranging from $50 \times 150$ to $300 \times 900$ grid points. The linear growth and nonlinear saturation is well represented from about 100 grid points in the longitudinal direction. The saturation marks the time when field line tension is able to locally overcome the centrifugal motion associated to the vortical motion, and coincides with a maximum reached by the cross-stream kinetic (and thus magnetic) energy, as shown in Fig. 3. At this point, a structure similar to the classical Kelvin's cat's eye (of pure hydrodynamics) is formed, enriched by local strands of strongly amplified magnetic fields. This also leads to magnetic reversals, so that the saturated structure is subsequently rapidly disrupted due to magnetic reconnection in fully resistive MHD. For later times, this structure survives for a long time, and the role of the magnetic field is to enhance its slow dissipation.

We have compared the time evolution of the three unstable $M_{\mathrm{s}}=1, M_{\mathrm{A}}=7$ jet profiles $(\operatorname{Pr} 1, \operatorname{Pr} 2$, and $\operatorname{Pr} 3)$ when initially perturbed with a varicose/sinuous mode. In accord with the linear stability results, the varicose mode is dominated by its sinuous counterpart, not only in terms of linear growth rate but also nonlinearly reaching a lower maximum perturbed energy, in agreement with Min (1997a,b). For example, the maximum perturbed energy reached in the varicose $\mathrm{KH}$ instability is 10 percent lower compared to the sinuous one for the $\operatorname{Pr} 1$ case, while for $\operatorname{Pr} 3$ it becomes negligible. The latter agrees with the linear finding that narrower jets have suppressed varicose growth rates, and are more unstable to sinuous 

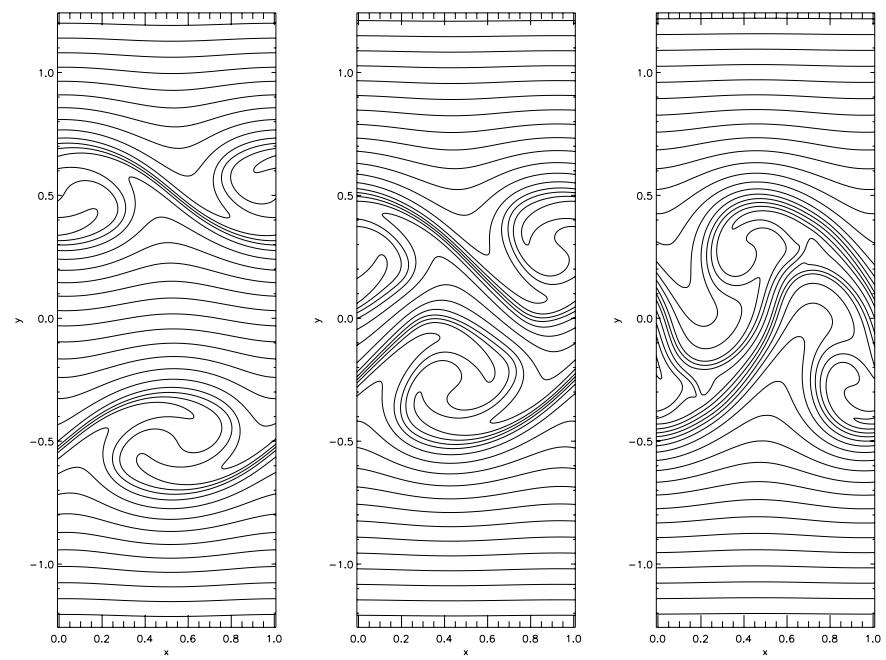

Fig. 4. Magnetic field lines at saturation $(t=3)$ for the three profiles ( $\operatorname{Pr} 1, \operatorname{Pr} 2$, and $\operatorname{Pr} 3$, from left to right respectively) initially perturbed with a sinuous mode. The physical case investigated is for $M_{\mathrm{s}}=1$, and $M_{\mathrm{A}}=7$. Only a part with $y$ in the range [-1.25:1.25] of the full computational domain is shown.

perturbations. The results showing the magnetic field deformation of sinuous $\mathrm{KH}$ instabilities are illustrated in Fig. 4, for a time close to saturation when the $\mathrm{KH}$ instability is well developed. One can easily observe that the $\mathrm{KH}$ vortices of the upper layer strongly interact with the vortices of the lower layer for the $\operatorname{Pr} 3$ case, contrary to the broad $\operatorname{Pr} 1$ jet configuration. In fact, the evolution of the $\mathrm{KH}$ modes for the "thick" jet $(\operatorname{Pr} 1)$ is very similar to results obtained for a single shear flow layer (Paper I) in the disruptive regime, as the two layers evolve quasi-independently. For the $\operatorname{Pr} 2$ profile, an interaction between the two layers occurs also before the saturation, somewhat weaker than in the $\operatorname{Pr} 3$ case. After the nonlinear saturation, the disruption of the vortices is illustrated in Fig. 5 for $\operatorname{Pr} 1$ and $\operatorname{Pr} 3$. Later, a relaxed state is reached, shown in Fig. 6. For the broad jet the quasi-steady laminar end-state consists of two enlarged (in the cross-stream direction) flow shear layers of heated and lower density plasma, encompassing the central jet core that is nearly unaffected. This is different for Pr3, as the whole initial flow profile is dramatically altered as its energy is effectively converted. The final relaxed states share some common properties in wide and narrow jet configurations: the velocity and magnetic field vectors end up nearly aligned everywhere and the longitudinal kinetic energy, which feeds the instability, has been strongly reduced compared to its initial level. In Fig. 7, we plotted the final and initial longitudinal component of the jet velocity profiles $V_{x}(y)$ at a given $x$ location, for the three cases. This confirms the above results, with the maximum value of the velocity nearly unaffected for $\operatorname{Pr} 1$, and decreased by a factor of order 2 for Pr3. We can quantify the disruptive effect by measuring the reduction of the normalized longitudinal kinetic en$\operatorname{ergy} \delta E_{k, x}=\left[E_{k, x}\left(t_{\mathrm{f}}\right)-E_{k, x}(0)\right] / E_{k, x}(0)$ between the final relaxed state obtained at $t=t_{\mathrm{f}}=20$ and the initial state at $t=0$. The results are plotted in Fig. 8 as a function of $M_{\mathrm{A}}$ for $M_{\mathrm{S}}=1$ and all three velocity profiles. In the latter figure, one can easily distinguish the three nonlinear regimes mentioned and defined
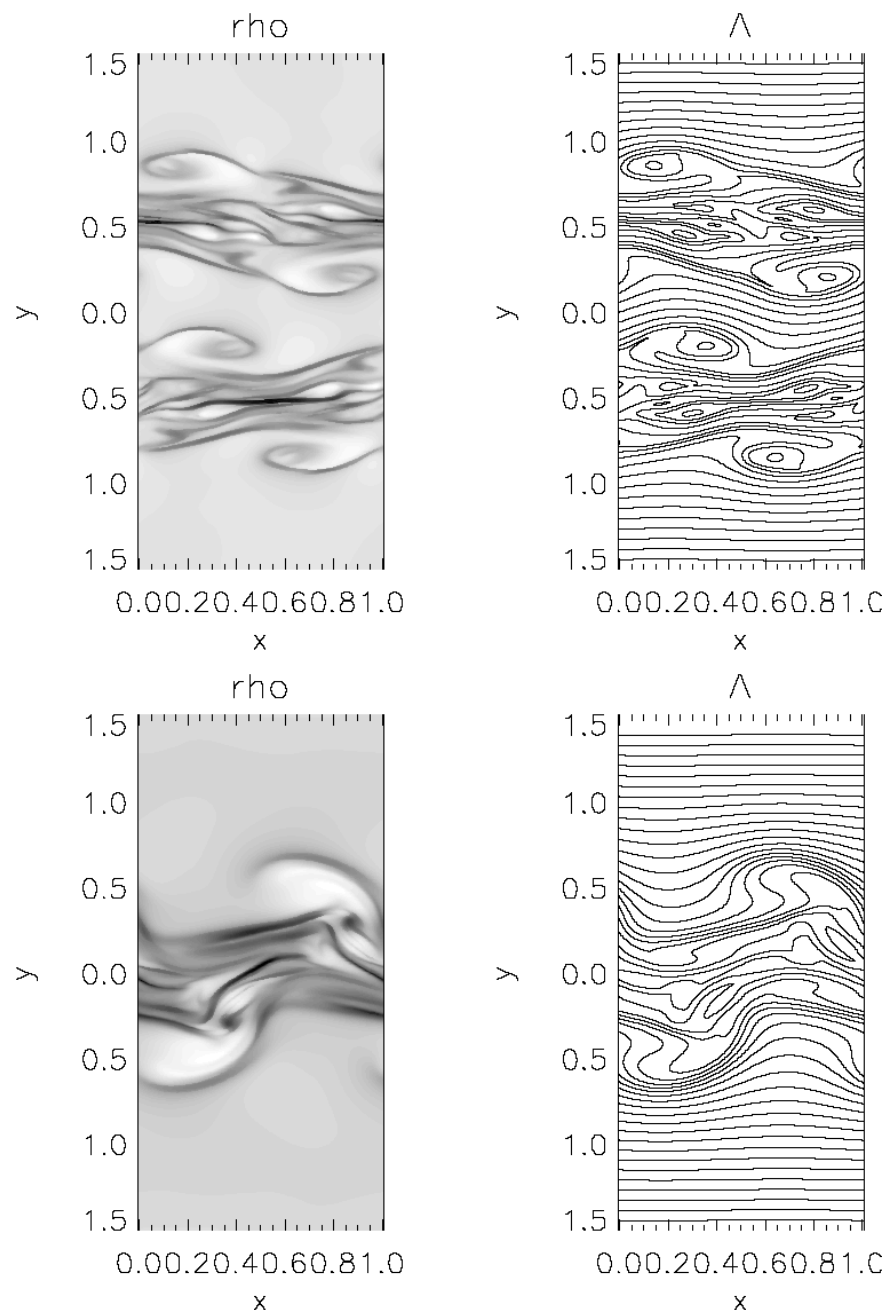

$0.00 .20 .40 .60 .81 . C$

$x$

A

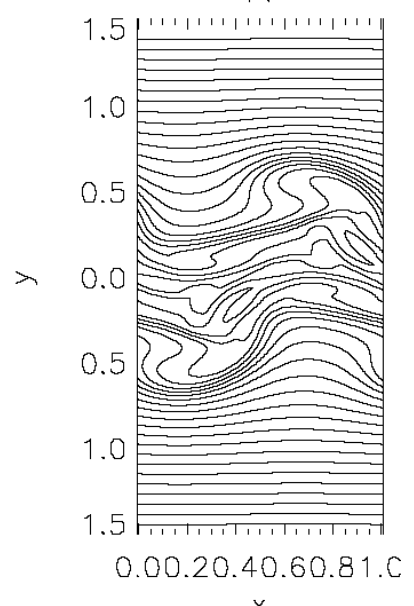

Fig. 5. Grey-scale images of the density distribution (left panels) in the $x-y$ plane, and corresponding magnetic field lines (right panels), during the disruption at $t=5$. $\operatorname{Pr} 1$ (top panels) and $\operatorname{Pr} 3$ (bottom panels) cases perturbed with a sinuous mode, for $M_{\mathrm{s}}=1$, and $M_{\mathrm{A}}=7$ are shown. Dark regions for correspond to low density values.

for a single shear flow layer in Paper I. The reduction of energy $\delta E_{k, x}$ increases for $M_{\mathrm{A}}$ in the range [2:4], reaches a plateau with a quasi-constant value for the disruptive regime, and decreases when $M_{\mathrm{A}} \gtrsim 30$ when we enter the modified hydrodynamic regime. The disruptive effect in terms of its maximum kinetic energy reduction is twice as high for $\operatorname{Pr} 3$ (70 percent) as for $\operatorname{Pr} 1$ (33 percent).

We have also investigated the supersonic regime using a slightly supersonic Mach number $M_{\mathrm{s}}=1.4$. The results are not shown because they are very similar to the transonic case, enriched by the formation of shocklet structures that are weak transient fast magnetosonic shocks (similar as obtained for a single shear flow layer in Paper I). For higher supersonic flows, it is not possible to investigate individual modes nonlinearly, because the initial perturbations ensure that "body" modes become unstable as well (see Fig. 2). 

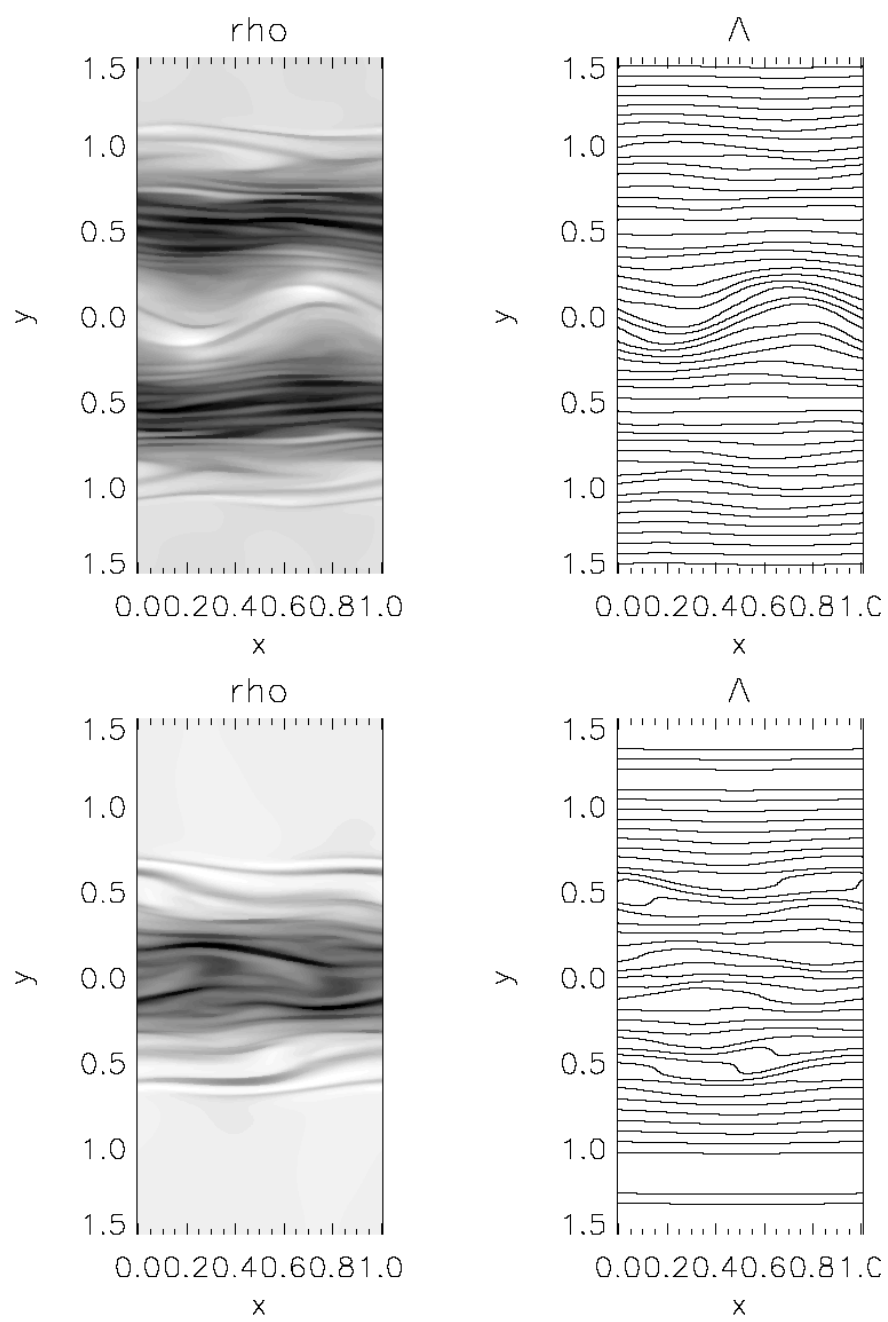

$0.00 .20 .40 .60 .81 . \mathrm{C}$

$\mathrm{x}$

$\wedge$

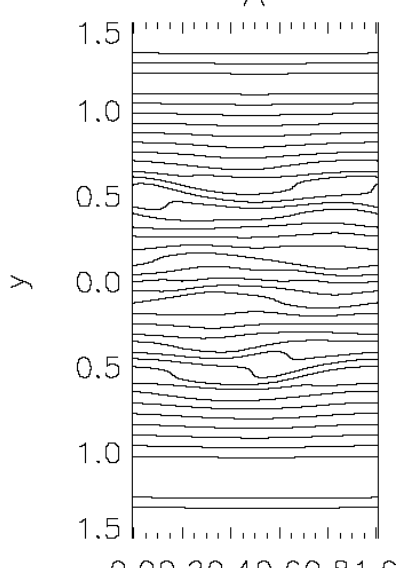

Fig. 6. Same as Fig. 5, but for the end of the simulations at $t=20$.

\subsection{Extended simulation domains}

We now consider simulations in a large spatial domain, typically $L_{x}=10$ for the periodic longitudinal length, allowing for roughly ten wavelengths of the most unstable mode. The lateral boundaries are placed at $y= \pm L_{y}= \pm 4$. We have used both VAC and AMRVAC with a spatial resolution of $800 \times 800$ grid points, up to effective resolutions of $1600 \times 1600$. More explicitely, this is achieved in AMRVAC simulations with a base resolution of $200 \times 200$ that is sufficient to resolve the initial vorticity thickness, and three finer levels of refinement. The choice of the spatial refinement strategy in such grid-adaptive simulations is important. In our simulations, we have checked for selected runs that AMR runs reproduce (much more efficiently in computational resources) results obtained with VAC for the same physical case. The system is perturbed with white noise.

Figure 9 shows density snapshots taken at three different times for a transonic broad $\operatorname{Pr} 1$ flow with $M_{\mathrm{A}}=7$. A largescale coalescence effect occuring early on each jet boundary is clearly visible. Indeed, the initial KH vortices (10 approximately, as one can see on the top panel) are able to pair/merge on each layer, leading to laterally enlarged structures (middle panel). This is in exact agreement with the coalescence mech-
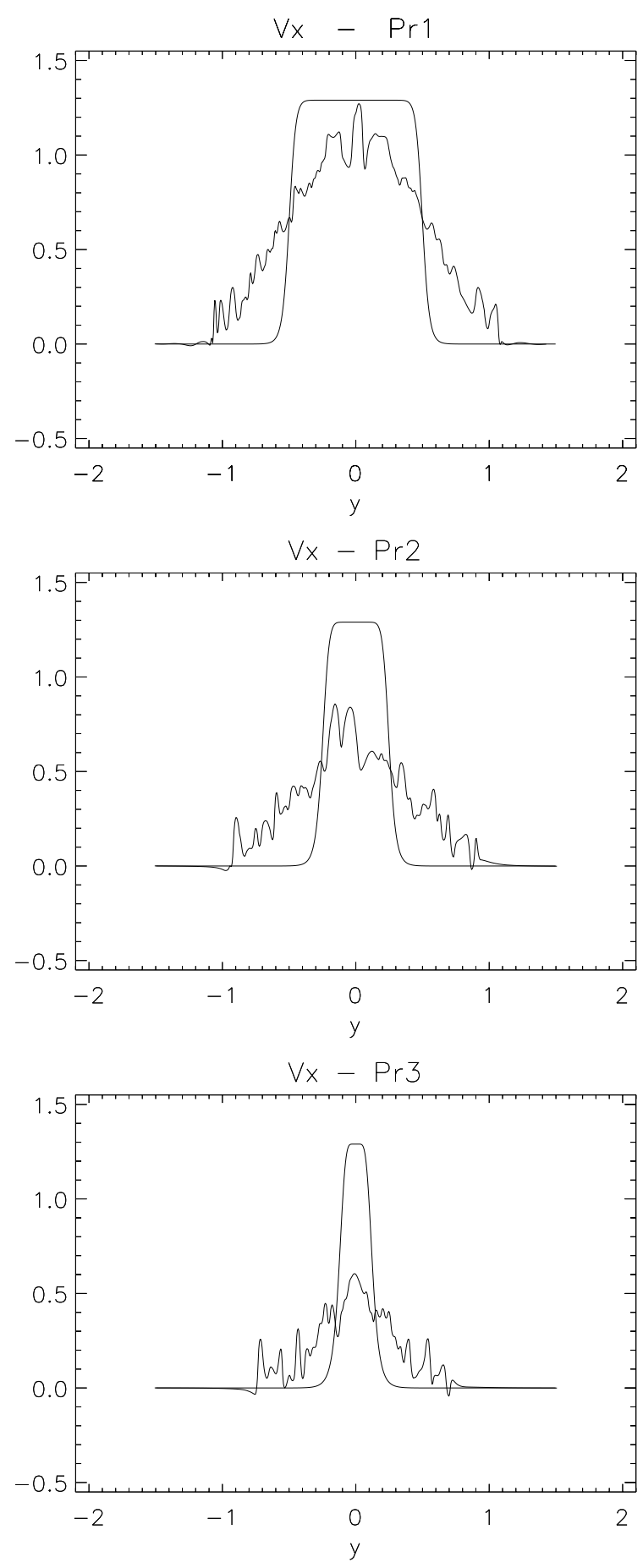

Fig. 7. One dimensional cut (at $x=0.5$ ) of the longitudinal velocity component $V_{x}(y)$, obtained at the end of the simulation for $\operatorname{Pr} 1$ (top), $\operatorname{Pr} 2$ (middle), and Pr3 (bottom) cases. For comparison, the initial longitudinal velocity component is also shown.

anism previously identified for a single shear flow layer in Paper I. During this pairing/merging mechanism the vortices tend to be partially disrupted due to magnetic reconnection events. However, the merged bigger vortices eventually force a strong interaction between the two edge layers, leading to a strong final disruption. In Fig. 10, the same flow parameters now characterize a narrow $\operatorname{Pr} 3$ slab jet: here there is a strong similarity between the extended and the short domain 


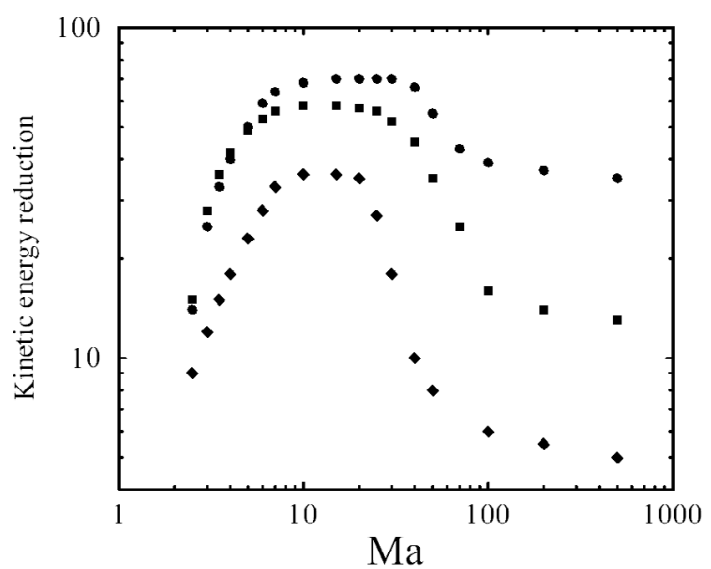

Fig. 8. Kinetic energy reduction (in percentage) $100 \times \delta E_{k, x}$ as a function of the Alfvén Mach number $M_{\mathrm{A}}$, for the three transonic $\left(M_{\mathrm{s}}=1\right)$ flow profiles $\operatorname{Pr} 1$ (diamonds), $\operatorname{Pr} 2$ (squares), and $\operatorname{Pr} 3$ (circles).

run, $L_{x}=10$ versus $L_{x}=1$, as the vortices of opposing edges interact immediately prior to the saturation (compare top panel of Fig. 10 with the bottom panels of Fig. 5), with a disruption ensuing just after. Pairing/merging events on individual layers are absent for this configuration. In Fig. 11 the cross-stream kinetic energy $E_{k, y}$ as a function of time is compared for the two profiles $\operatorname{Pr} 1$ and $\operatorname{Pr} 3$. For the broad jet $\operatorname{Pr} 1$, the large-scale coalescence occurs for a long time building up in perturbed energy, with an overall saturation much later compared to $\operatorname{Pr} 3$. The latter narrow jet shows pronounced kink deformations at the time of saturation, in agreement with the linear stability results. Contrary to non-extended configurations having $L_{x}=1$, the overall saturation level for the two profiles are comparable, thanks to the pairing/merging process of the $\operatorname{Pr} 1$ case. If we measure the kinetic energy reduction due to the disruptive effect, the results give a value close to 70 percent for all flow profiles, from broad to narrow jets.

Turning to supersonic flows having $M_{\mathrm{s}}=3$, the same Alfvénic Mach number translates in a plasma beta parameter $\beta=6.5$, an order of magnitude reduction to the transonic case. We again show density snapshots in Figs. 12 and 13, for the $\operatorname{Pr} 1$ and $\operatorname{Pr} 3$ flow profiles respectively. Essential differences are evident compared to the transonic regime. First, the dominant surface mode that deforms the interface is characterized by flattened vortex-like structures for the broad $\operatorname{Pr} 1$ jet and by sinusoidal oscillations of the whole flow for the narrow supersonic jet (top panels of the two figures). Second, additional deformations inside the jet flow are most clearly seen for the broad jet, attributable to internal body modes. Both supersonic jet flows are seen to induce strong compressive perturbations in the external medium, steepening rapidly into strong shocks. This, together with the pronounced sinuous deformation of the narrow jet, is in very good agreement with the linear stability results. The linear results for the broad supersonic jet (not shown) indicate the presence of many body modes with significant density variation in the external medium. Note that we deduce from the periodicity of the features observed in these two figures that we have only 5 or 6 linearly unstable wavelengths, contrary to the transonic regime where 10 were present. This corresponds
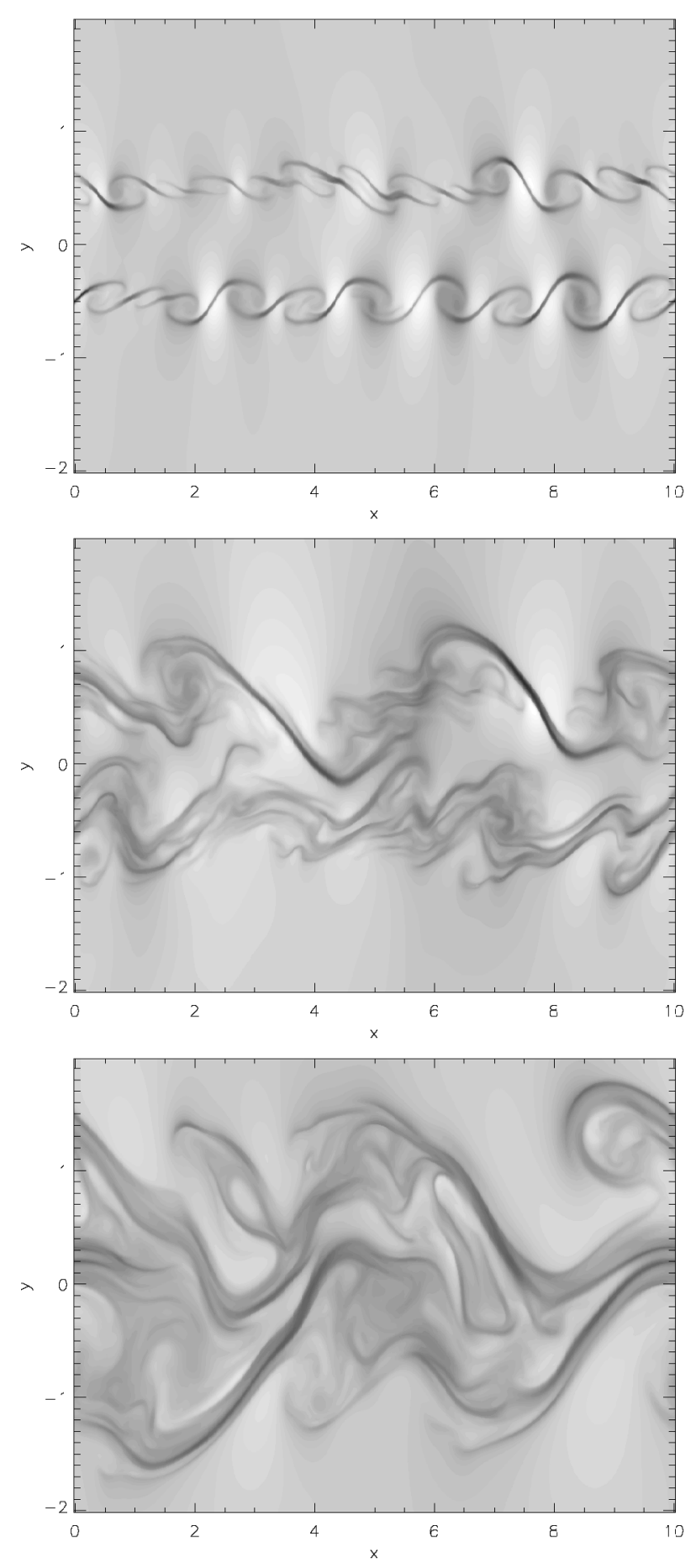

Fig. 9. Grey-scale images of the density distribution obtained at three different times ( $t=7,13,20$, from top to bottom respectively) for a $\operatorname{Pr} 1$ profile in the large computational domain. The physical case investigated is for $M_{\mathrm{s}}=1$, and $M_{\mathrm{A}}=7$. A linear grey-scale is used with density values ranging from 0.65 to 1.15 . Note that only a part with $y$ in the range [-2:2] of the full domain is shown.

well to the shift to longer wavelengths for the most unstable mode observed in the linear analysis. This shift towards higher longitudinal wavelengths as the fast Mach number increases is quantified also in Baty (2005). At later times, some large-scale coalescence effect occurs before saturation for $\operatorname{Pr} 1$ (see second panel of Fig. 12), while the narrow supersonic jet shows a sud- 

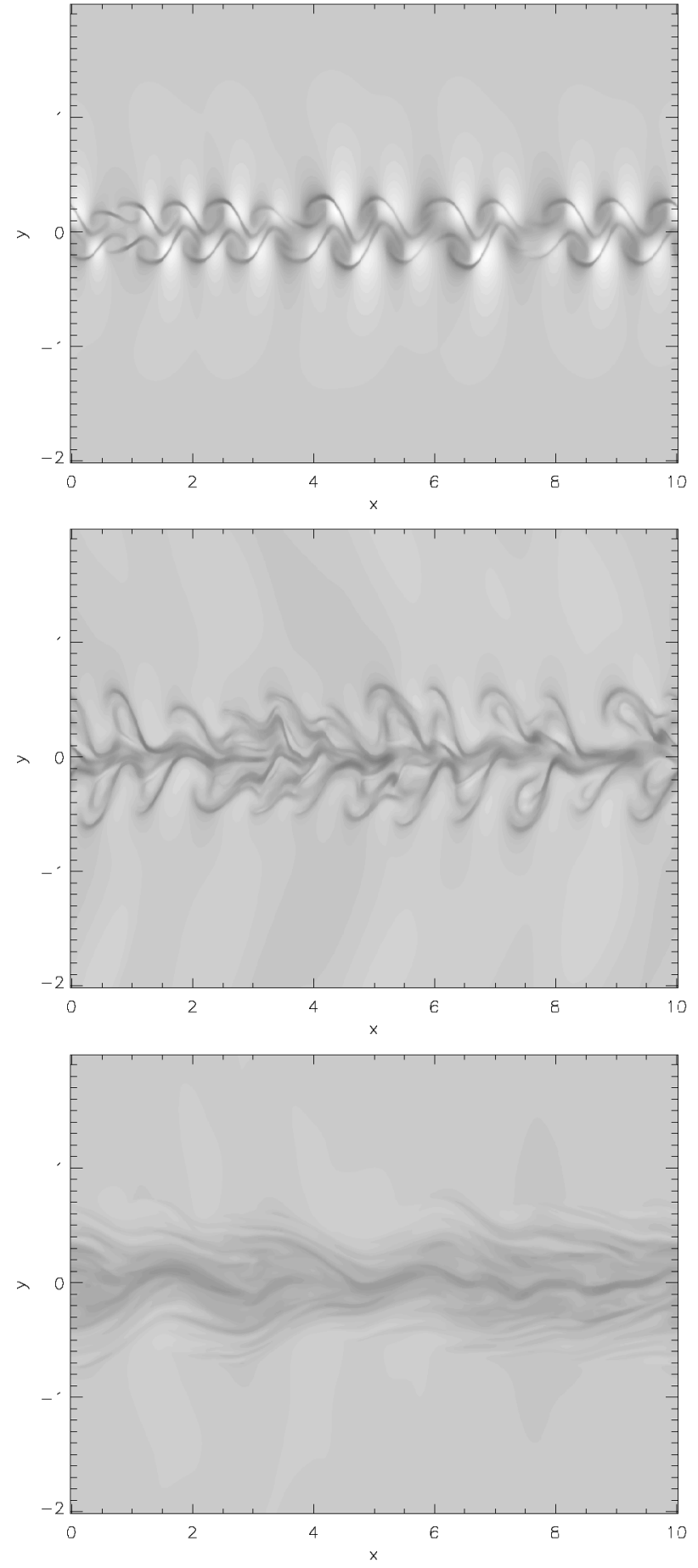

Fig. 10. Same as Fig. 9, but for the $\operatorname{Pr} 3$ case. The snapshots correspond to $t=5,8,18$ from top to bottom respectively.

den, shock-dominated disruption after a few time units. The final states obtained (not shown) are again characterized by very low residual velocities, with the jet kinetic energy efficiently converted to thermal energy. The measure of the kinetic energy reduction introduced in the previous section, gives a value close to 80 percent for the two profiles.

In order to quantify the effect of the flow profile on the energy cascade direction in somewhat more detail, we examined spatial Fourier spectra. Since the configurations considered in

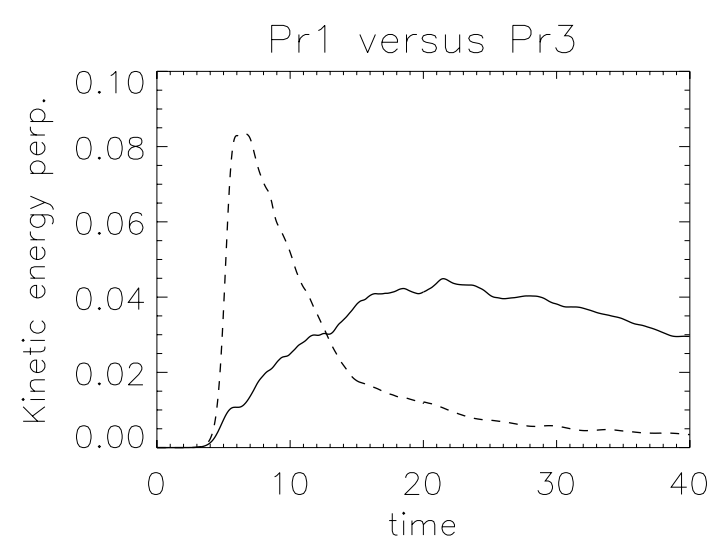

Fig. 11. Time evolution of the perpendicular kinetic energy $E_{k, y}$ for $\operatorname{Pr} 1$ (plain line) and $\operatorname{Pr} 3$ (dashed line) cases in the large computational domain. The values are normalized using the initial background kinetic energy of the flow $E_{k, x}(t=0)$. The physical case investigated is for $M_{\mathrm{s}}=1$, and $M_{\mathrm{A}}=7$.

this study are periodic in the $x$-direction only, the flow can be assumed statistically homogeneous only in the longitudinal direction. Spatial Fourier transforms are consequently computed in the $x$-direction. For example, the one-dimensional longitudinal energy spectrum of the longitudinal velocity $V_{x}$ is given by

$E\left(k_{x}\right)=\frac{1}{2 L_{y}} \int_{-L_{y}}^{L_{y}} U^{2}\left(k_{x}, y\right) \mathrm{d} y$,

where $U\left(k_{x}, y\right)$ is the $y$-dependent longitudinal Fourier transform of the $V_{x}$ velocity,

$U\left(k_{x}, y\right)=\frac{1}{L_{x}} \int_{0}^{L_{x}} V_{x}(x, y) \exp \left(-\mathrm{i} k_{x} x\right) \mathrm{d} x$.

The resulting longitudinal energy spectra are plotted in Figs. 14 and 15 for transonic $\operatorname{Pr} 1$ and $\operatorname{Pr} 3$ cases respectively, at different times. For both profiles, the spectrum is clearly dominated by the contribution of $n \approx 10$ (where $k_{x}=2 \pi n / L_{x}$ ) modes at early times, confirming that roughly 10 linearly unstable (dominant) wavelengths are initially growing. This energy injected by the dominant linear modes is quickly transferred to lower mode numbers (longer wavelengths), signaling the inverse cascade. For the broad jet $\operatorname{Pr} 1$, this inverse cascade occurs very soon, as the system is still building up in perturbed energy: an $n=2$ mode already dominates before saturation at $t=13$. This clearly occurs in correspondence with the pairing/merging events already reported previously. For the narrow jet $\operatorname{Pr} 3$, close to the saturation time (i.e. $t=8$ ) the subharmonic $n=5$ has grown to an energy level comparable to $n=10$, and it dominates the spectrum at later times, e.g. during the disruption at $t=18$. Hence, an inverse cascade process is also at work for $\operatorname{Pr} 3$ despite the absence of pairing/merging process, starting later in time during the disruption. The final end-states shown in spectral energy content at $t=40$ for both profiles are dominated by the largest longitudinal wavelength available in the domain. Analysing the evolution of the slope dependence of the spectra with $n$, for $10 \lesssim n \lesssim 100$ the energy spectrum for this intermediate wavenumber range settles on a $k^{p}$ law with a index $p$ value close to -4 whatever the velocity profile. For 

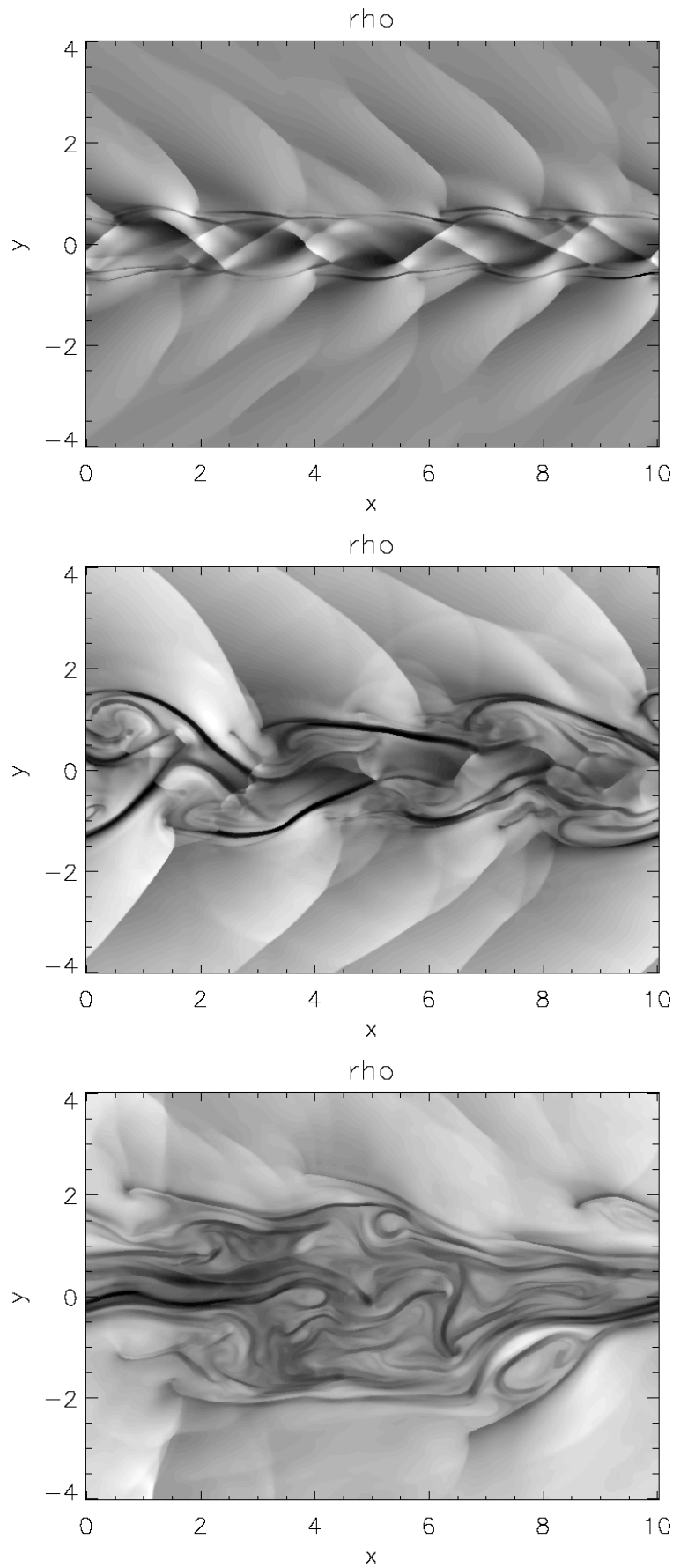

Fig. 12. Grey-scale images of the density distribution obtained at three different times $(t=10,15,25)$ for a $\operatorname{Pr} 1$ profile in the large computational domain. The physical case investigated is a supersonic jet having $M_{\mathrm{s}}=3$, and $M_{\mathrm{A}}=7$. A linear grey-scale is used with density values ranging from 0.15 to 1.95 .

the range $1<n \lesssim 10$ that corresponds to a domain of wavelengths that are larger than the linearly fastest growing one, a different exponent with a value between $k^{-1}$ and $k^{-2}$ is found. The numerical dissipation influences the spectral dependence significantly beyond $n \gtrsim 100$ at the present resolution. Finally, note that despite the presence of shocks, similar results and conclusions are obtained from the analysis of energy spectra for supersonic $\operatorname{Pr} 1$ and $\operatorname{Pr} 3$ cases.
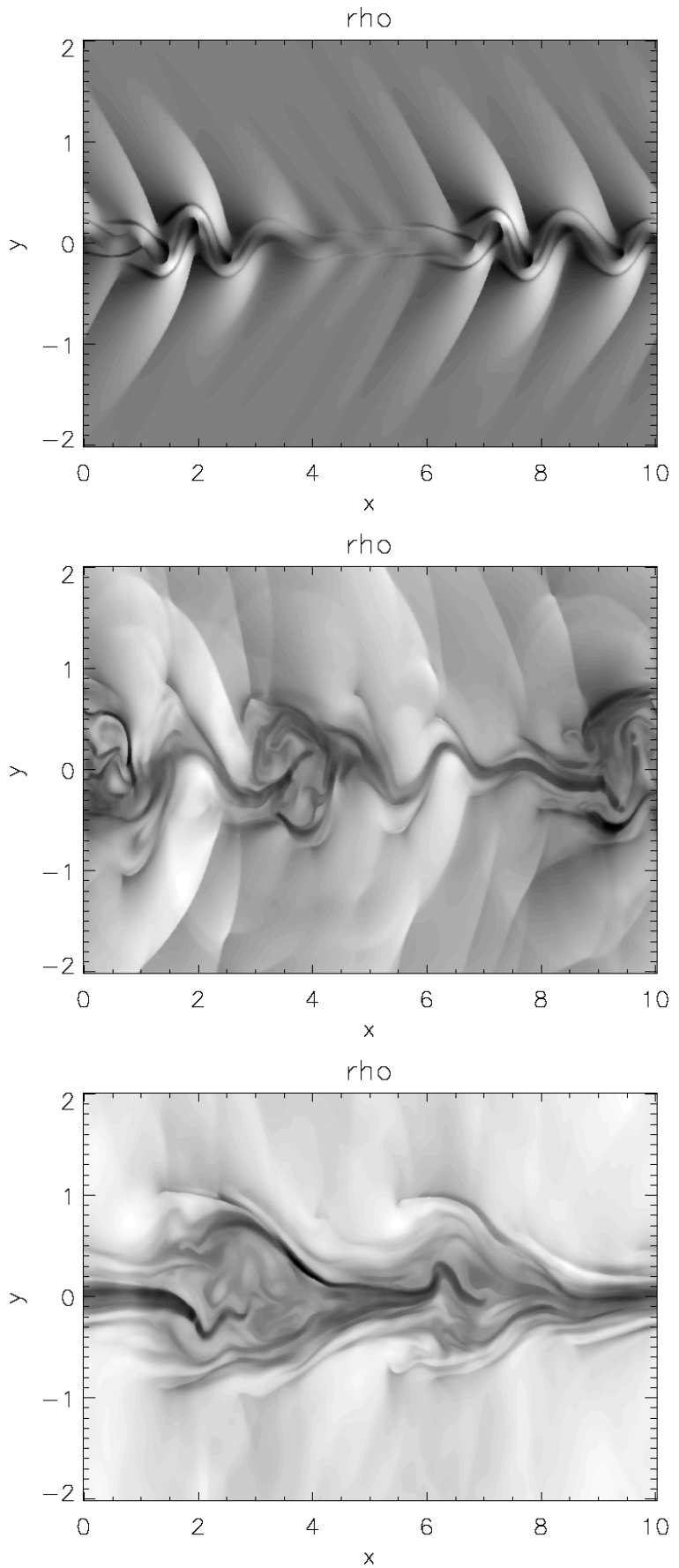

Fig. 13. Same as Fig. 12, but for the $\operatorname{Pr} 3$ case. The images correspond to $t=4,6,10$ from top to bottom respectively.

The $p=-4$ index of the intermediate $k$-range follows closely the slope law between $k^{-4}$ and $k^{-3}$ obtained by Lesieur et al. (1988), in 2D purely hydrodynamic numerical simulations of extended unstable shear flow layers. This is not surprising, as for the parameters characterizing our jets, the kinetic energy is dominant over other energy forms (in fact the magnetic energy is dominant only temporarily and locally at saturation). Miura (1999) also reported a power law exponent of -3.89 in two-dimensional transonic shear layers with a magnetic field purely perpendicular to the shear flow. The weakly magnetized planar jets studied here suggest this to be a rather 

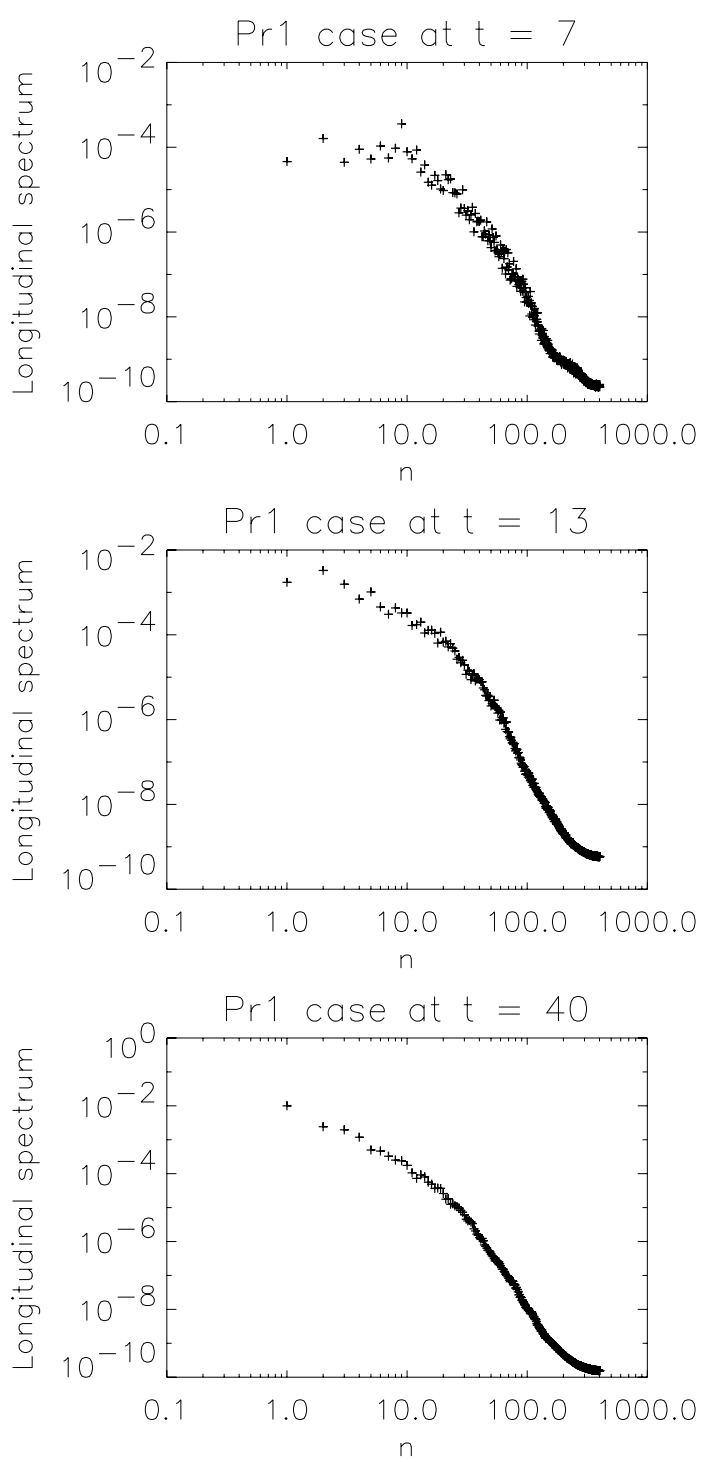

Fig. 14. Longitudinal spectrum $E\left(k_{x}\right)$ obtained for the transonic $\operatorname{Pr} 1$ case, with $n$ labelling the longitudinal wavenumber $k_{x}$ via the relation $k_{x}=2 \pi n / L_{x}$.

general characteristic. One may note that in freely decaying, homogeneous 2D hydrodynamic turbulence energy spectral indices of $k^{-3}$ signal the simultaneous operation of an inverse energy cascade together with a direct cascade in enstrophy (Biskamp 1993; Rutgers 1998).

As a final example of the dynamics of extended domain jet simulations, we performed a simulation with parameters characteristic of the modified hydrodynamic regime for a narrow jet, i.e. with $M_{\mathrm{s}}=1$ and $M_{\mathrm{A}}=50$. The magnetic field is very weak, as the plasma $\beta=3000$. Figure 16 illustrates one snapshot of the density patterns obtained. One can see how well developed vortices originating on each side of the jet couple and survive as pairs for quite long times. This coupling of counterrotating vortices without merging, is known from pure 2D hydrodynamics as Batchelor coupling (Lesieur et al. 1988). These vortex pairs tend to leave the jet core without being further disrupted, and this is only slightly modified by very weak and lo-
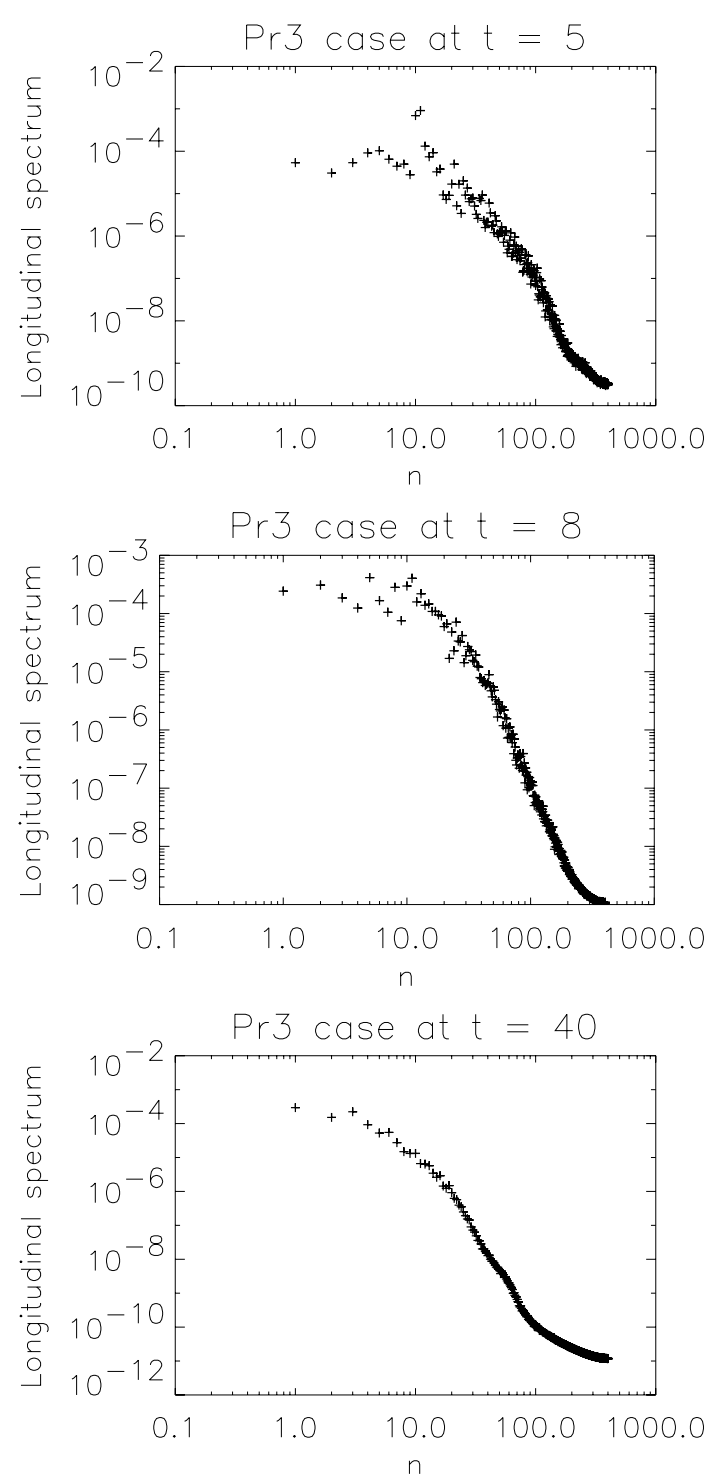

Fig. 15. Same as Fig. 14, but for the $\operatorname{Pr} 3$ case.

calized magnetic effects, causing the vortices to be surrounded by narrow density strands of depleted material.

\section{Nonlinear results: 3D cylindrical jets}

The main differences expected in $3 \mathrm{D}$ versus $2 \mathrm{D}$ come from the existence of unstable modes that are absent in 2D and are typical of a 3D cylindrical geometry. Indeed, it is known from stability theory (see Birkinshaw 1991 for example) and also from numerical experiments (Bodo et al. 1998; Hardee 2004, and references therein) that a wealth of body/surface modes are linearly unstable in supersonic jets (see also Fig. 2). The growth rates for $m>1$ can be comparable or even greater than the $m=1$ one when the Mach numbers are much greater than unity. We present few selected 3D simulations with a moderate, fixed spatial grid resolution in the cross-stream plane of $100 \times 100$ and with streamwise resolution up to 250 . The domain dimension was taken up to $L_{z}=5$, and lateral boundaries typically at unit distance. This corresponds to rather reduced resolution when compared to our $2 \mathrm{D}$ runs, as the 


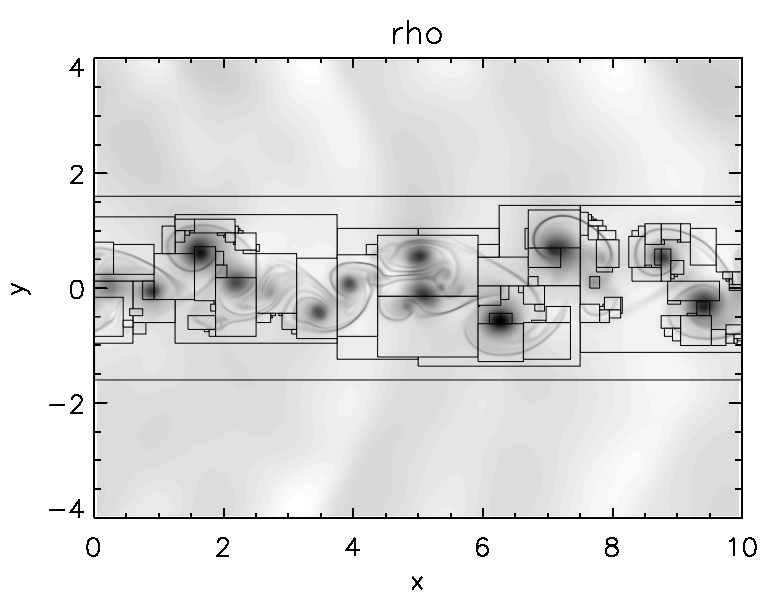

Fig. 16. Grey scale images of the density distribution obtained for a transonic $\operatorname{Pr} 3$ flow profile with $M_{\mathrm{A}}=50$. This figure is taken at a time after the saturation. Also indicated is the location of the finest level grids in this grid-adaptive simulation.

computational cost became prohibitively high in $3 \mathrm{D}$ for long term simulations. Nevertheless, this resolution is sufficient to compare the morphological deformations induced by the development of the $\mathrm{KH}$ instabilities in 3D versus $2 \mathrm{D}$.

Starting with a transonic $M_{\mathrm{s}}=1$ superAlfvénic $M_{\mathrm{A}}=7$ configuration, we investigated both a wide $(\operatorname{Pr} 1)$ and narrow (Pr3) jet. The results show large-scale deformations, that resemble the corresponding 2D cases, and that can be clearly attributed to the development of the $m=1$ mode for early times. This is discernable in 2D longitudinal cuts, which follow their 2D counterparts. Additionally, we observe that structures due to the development of $m>1$ modes contribute to the deformation of the flow. As an example, in the transverse cut shown in Fig. 17 (top panel) an elliptical deformation is present close to saturation for the transonic $\operatorname{Pr} 1$ case, thus corresponding to the growth of an $m=2$ mode that is linearly unstable. The latter figure could be contrasted to Fig. 9a shown in Baty \& Keppens (2002), where a similar uniformly magnetized 3D case with only an $m=1$ surface mode perturbed is computed. For later times, a disruption occurs like in $2 \mathrm{D}$, on a comparable timescale. However, small-scales structures (see bottom panel of Fig. 17) strongly contribute to the deformation of the flow. This reflects that the direct cascade towards high wave numbers is more efficient in $3 \mathrm{D}$ than in $2 \mathrm{D}$ due to more possibilities in the nonlinear interactions. This is in agreement with results obtained by Bodo et al. (1998), showing that the flow disruption in purely hydrodynamic simulations is more turbulent in 3D versus 2D. Weak evidence for the existence of an inverse cascade is found from spectra taken from 2D longitudinal cuts. The case of a narrow jet shows the expected faster helical disruption, with early mode-mode interactions taking place. The final endstate is more turbulent in terms of density and velocity fluctuations, but the broadening of the jet profile roughly agrees with its $2 \mathrm{D}$ analogue.

An extended $\left(L_{z}=5\right)$ supersonic $M_{\mathrm{s}}=3$ jet at the same Alfvén Mach number but of intermediate radial extent ( $P r 2)$ was simulated up to time $t=20$, corresponding to twice the saturation time. The disruption is now again shock-dominated,
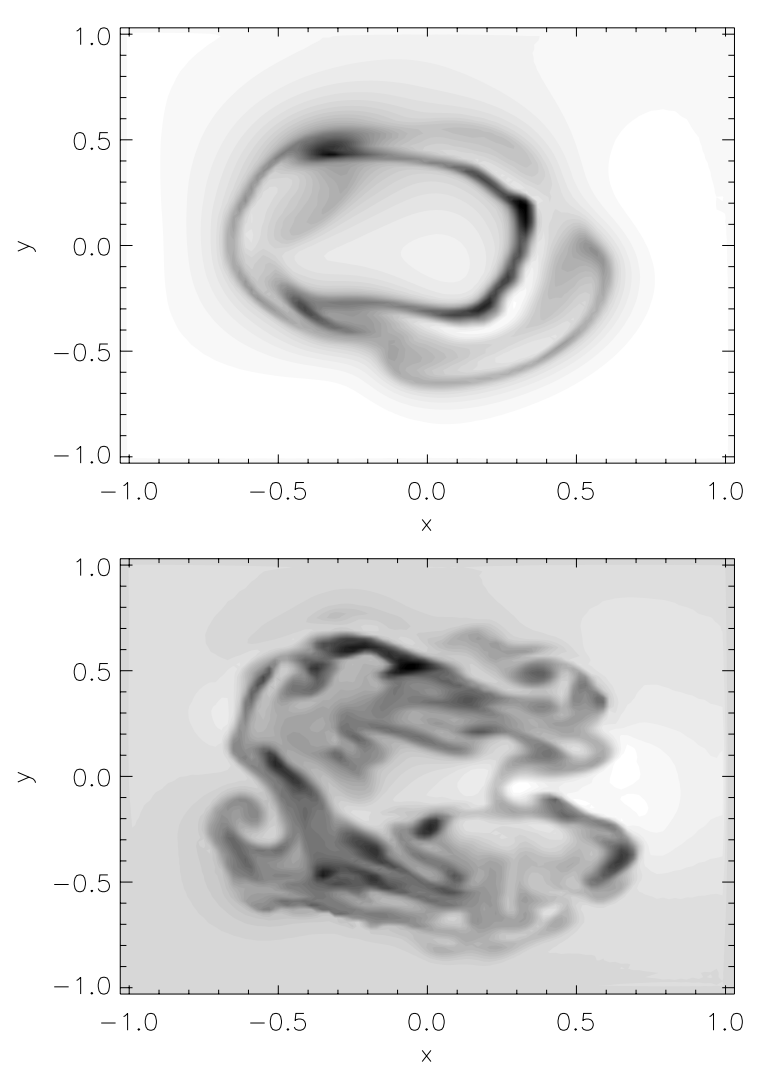

Fig. 17. Grey scale images (2D cut obtained at $x=2$ ) of the density distribution obtained for a 3D run of an unstable transonic $\operatorname{Pr} 1$ flow profile with $M_{\mathrm{A}}=7$. The top panel corresponds to a time $t=8$ close to saturation, and the bottom panel to a time $t=13$ during the disruption.

with external shocks extending to the lateral domain boundaries. We show in Fig. 18 an isosurface of the vorticity magnitude of order of the initial maximal value, combined with a cutting plane showing the pressure. The isosurface shows how several helical mode numbers contribute to the jet deformation. In the streamwise direction eventually one to two shock fronts emerge before and at saturation, and a rapid transition to a broadened, heated jet characterized by an Alfvén Mach number of about 1 follows. The longitudinal energy spectra for the axial velocity are initially dominated by modes with axial wavenumber $n=2$ and $n=3$, with eventual tendency to follow monotonically decreasing $k^{p}$ power law behavior. An exponent of $p=-4$ characterizes the intermediate wavenumber range in the strong disruption stage. Similarities are thus evident for all cases explored in 3D to those obtained in 2D, and the extended magnetized jet transits to a heated, low velocity structure. The central sonic mach number reached for all cases discussed above ends up significantly below unity, an order of magnitude reduction in the supersonic case.

\section{Summary and astrophysical relevance}

We can summarize our findings as follows. We have numerically studied the temporal development of $\mathrm{KH}$ instabilities that occurs in uniformly magnetized jets. We focus on transonic/supersonic flows embedded in a parallel magnetic field, 


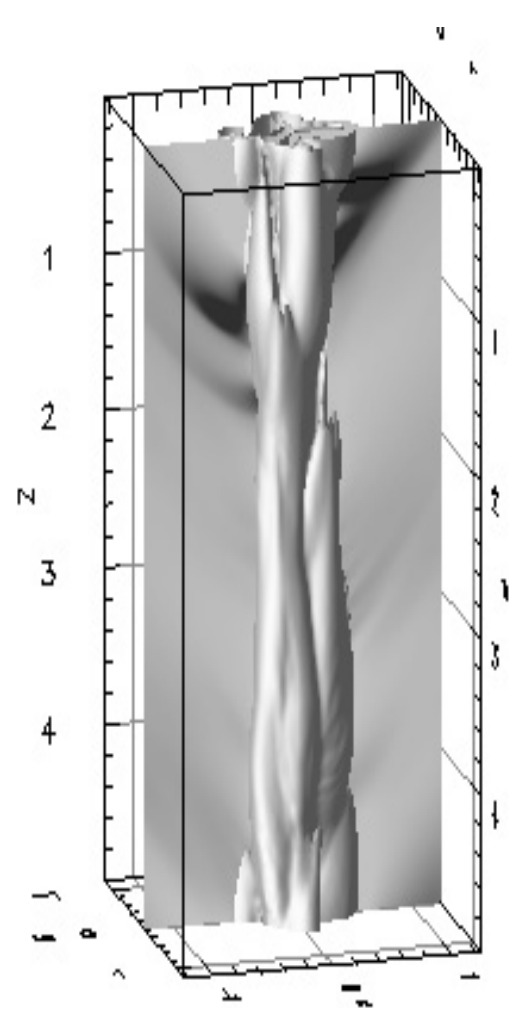

Fig. 18. An isosurface of vorticity magnitude, and a cross-cut showing the pressure, for a supersonic $M_{\mathrm{s}}=3$ superAlfvénic jet close to saturation at $t=9$.

with amplitudes weak enough to have a negligible effect on the linear stability. Nevertheless, the magnetic field strongly influences the nonlinear dynamics compared to pure hydrodynamics. We extrapolate previous studies made for individual modes (allowing only the growth of single structures corresponding to the linearly fastest longitudinal wavelengths) to extended 2D and 3D longitudinally periodic configurations.

In the nonlinear evolution of individual modes for 2D transonic flows, we quantified the ability of the jet flow to survive $\mathrm{KH}$ instabilities. The width of the flow profile is crucial in this respect. Indeed, for jets having a radius much larger than the vorticity thickness, the disruption of $\mathrm{KH}$ vortices due to magnetic reconnection is only able to enlarge the interface leaving the jet core almost unaffected. The fate of jets having large vorticity thickness of the order of the radius, is fundamentally different, as the whole jet is strongly affected and the major part of the initial kinetic energy of the background flow is now converted into other forms of energy. Second, the use of large spatial 2D domains has allowed us to explore the interaction of several longitudinal wavelengths of the linearly dominating modes in transonic/supersonic flows. A self organization process is observed in all simulations, that proceeds through an inverse cascade of energy in Fourier spectra. For a broad jet profile, this trend towards large scales proceeds early via pairing/merging of the $\mathrm{KH}$ structures, which in turn allows the system to reach a higher level of perturbation, compared to a similar simulation of an individual mode. For a narrow jet profile, the inverse cascade is observed later, after saturation and during the disruption stage. Overall, the kinetic energy reduction quantifying the disruption is similar for the different initial velocity profiles. For transonic flows $M_{\mathrm{s}}=1$, the kinetic energy reduction is roughly equal to 70 percent. However, supersonic flows additionally exhibit strong shocks developing inside the jet core, as a consequence of the nonlinear evolution of $\mathrm{KH}$ body modes. Moreover, rapid shock dominated transients characterize the disruption: the corresponding kinetic energy reduction can reach up to roughly 80 percent for $M_{\mathrm{s}}=3$.

Finally, 3D simulations of cylindrical jets show a similar phenomenological behaviour with respect to the cascade directions and flow disruption. This is not surprising as, in contrast to hydrodynamics, MHD is known to have similar physical properties in 2D versus 3D (Biskamp 1997). This is true for turbulence cascade directions, as well as for the energy spectra deduced from homogeneous turbulence theory. This reinforces the relevance and importance of our simulations in 2D geometry, where a much higher spatial resolution can be reached compared to 3D. There is a noticeable difference: the disruption is observed to be more turbulent in 3D due to the presence of typical 3D modes, which are either linearly unstable with a non-negligible growth rate, or which are driven by nonlinear couplings.

The issue of large-scale magnetized flow coherence and survival is fundamentally important for astrophysical jets. Indeed, the remarkable stability deduced from observations can not be reproduced by high-resolution hydrodynamic simulations (Bodo et al. 1995, 1998), even if density contrast (between the jet core and the external medium) and/or radiative effects (using well chosen cooling functions) can reduce the discrepancy (Downes \& Ray 1998; Micono et al. 2000; Stone et al. 1997). Some jets can probably achieve a partial stabilisation through different strategies. For example, a high Lorentz factor can help by significantly reducing the growth rate for relativistic jets (Hardee 2004), as well as the presence of an external magnetized wind in cases of YSO jets is found to be stabilizing against the disruptive modes (Hardee \& Rosen 2002). On the other hand, one can not ignore the presence of a largescale magnetic field, which is necessary for an efficient collimation of the flow. Thus, the solution of the problem could be also magnetic. Our results show that the nonlinear evolution of $\mathrm{KH}$ instabilities is strongly enriched by the presence of a magnetic field, allowing in particular magnetic reconnection events. However, jet flows disrupt quickly in MHD as well. Indeed, translating the typical disruption time obtained in our simulations into a disruption length (KH modes are known to be advected at approximately half the jet velocity), we get a characteristic length that is of order a few ten times the jet radius. This latter value has to be compared to observations showing that some jets are able to propagate over distances up to 1000 times their radial extent. The only way to magnetically stabilize jet configurations like those studied here, is to have a magnetic field component parallel to the flow of sufficiently strong amplitude to linearly stabilize the $\mathrm{KH}$ modes (i.e. for a parallel Alfvén Mach number $M_{\mathrm{A}} \lesssim 2$ ). Such strong magnetic fields are hard to reconcile with observations of astrophysical jets. On the other hand, there are observational indications as well as theoretical arguments in favour of a large-scale magnetic field exhibiting a helical geometry inside the jet. This may well 
change the results and conclusions presented here. Recent full three-dimensional similations have shown that, the presence of a significant azimuthal field component is crucial in maintaining a higher flow stability (Rosen et al. 1999). Moreover, the presence of a background helical field efficiently suppress the turbulent cascade towards small scales, thanks to the hoop force. Second, the nonlinear interaction between simultaneously growing $\mathrm{KH}$ and current-driven (due to the presence of an electrical current inside the jet core) modes can in fact aid in jet survival as demonstrated in Baty \& Keppens (2002). The latter study was done for a transonic flow of a longitudinally short periodic jet, and should be extended to larger simulation domains and to a wider range of Mach numbers. The interpretation of the results for extended fast jets with more realistic magnetic field configurations can benefit from earlier detailed numerical experiments on transmagnetosonic single shear flow layers in 2.5 and 3 dimensions with sheared magnetic fields (Jeong et al. 2000; Ryu et al. 2000).

Beside the issue of jet survival, internal instabilities in astrophysical jets are evoked to explain typical morphological structures seen in observations, presumably acting together with a concurrent mechanism based on intrinsic variability in the jet source (see review by Gouveia Dal Pino 2005, and references therein). This is the case for the chains of aligned knots observed in Herbig-Haro jets ejected from young stars (Thiele \& Camenzind 2002), as well as the wiggled structures seen in AGN jets (Nakamura \& Meier 2005). Second, they could partially explain the morphological dichotomy in observed extragalactic jets, between Fanaroff-Riley type I (FR I) and FR II jets.

Acknowledgements. This work was supported in part by the European Community's Human Potential Programme under contract HPRNCT-2000-00153, PLATON. R.K. did the work under the EuratomFOM Association Agreement and in the Computational Science programme "Rapid Changes in Complex Flow" funded by NWO-E, coordinated by J.P. Goedbloed. The numerical calculations were performed on the SGI Origin 3800 at CINES, Montpellier (France) and at supercomputing facilities sponsored by the "Stichting Nationale Computerfaciliteiten" (NCF) and NWO. H.B. thanks J. P. Goedbloed for his hospitality during his visit to the FOM institute. We also thank P. Comte for useful discussions, and the anonymous referee for suggestions that helped to improve the content of the paper.

\section{References}

Appl, S., \& Camenzind, M. 1992, A\&A, 256, 354
Baty, H. 2005, A\&A, 430, 9

Baty, H., \& Keppens, R. 2002, ApJ, 580, 800

Baty, H., Keppens, R., \& Comte, P. 2003, Phys. Plasmas, 10, 4661 (Paper I)

Biskamp, D. 1993, Nonlinear Magnetohydrodynamics (Cambridge Univ. Press)

Birkinshaw, M. 1991, in Beams and jets in Astrophysics, ed. P. A. Hugues (Cambridge Univ. Press), 279

Bodo, G., Massaglia, S., Rossi, P., et al. 1995, A\&A, 303, 281

Bodo, G., Rossi, P., Massaglia, S., et al. 1998, A\&A, 333, 1117

Brackbill, R. B., \& Barnes, D. C. 1980, J. Comput. Phys., 35, 426

Casse, F., \& Keppens, R. 2004, ApJ, 601, 90

Collela, P., \& Woodward, P. R. 1984, J. Comput. Phys., 54, 174

Downes, T. P., \& Ray, T. P. 1998, A\&A, 331, 1130

Ferrari, A. 1998, Annual Rev. Astrophys., 36, 539

Gabuzda, D. C., Murray, E., \& Cronin, P. 2004, MNRAS, 351, 89

Gouveia Dal Pino, E. M. 2005 [arXiv: astro-ph/0406319]

Hardee, P. E. 2004, Ap\&SS, 293, 117

Hardee, P. E., \& Rosen, A. 2002, ApJ, 576, 204

Hardee, P. E., Cooper, M. A., Norman, M. L., \& Stone, J. M. 1992, ApJ, 399, 478

Harten, A. 1983, J. Comput. Phys., 49, 357

Jeong, H., Ryu, D., Jones, T. W., \& Frank, A. 2000, ApJ, 529, 536

Keppens, R., Nool, M., Tóth, G., \& Goedbloed, J. P. 2003, Comput. Phys. Comm., 153, 317

Lesieur, M. 1997, Turbulence in Fluids, 3rd ed. Kluwer

Lesieur, M., Staquet, C., Le Roy, P., \& Comte, P. 1988, J. Fluid Mechanics, 192, 511

Micono, M., Bodo, G., Massaglia, S., et al. 2000, A\&A, 360, 795

Min, K. W. 1997a, ApJ, 482, 733

Min, K. W. 1997b, MNRAS, 285, 191

Miura, A. 1999, J. Geophys. Res., 104, 295

Nakamura, M., \& Meier, D. L. 2005, ApJ, 617, 123

Nijboer, R. J., van der Holst, B., Poedts, S., \& Goedbloed, J. P. 1997, Comp. Phys. Comm., 101, 39

Pushkarev, A. B., Gabuzda, D. C., Vetukhnovskaya, Y. N., \& Yakimov, V. E. 2005, MNRAS, 356, 859

Ray, T. P., Musclow, T. W. B., Axon, D. J., et al. 1997, Nature, 385, 415

Rosen, A., Hardee, P. E., Clarke, D. A., \& Johnson, A. 1999, ApJ, 510, 136

Rutgers, M. A. 1998, Phys. Rev. Lett., 81, 2244

Ryu, D., Jones, T. W., \& Frank, A. 2000, ApJ, 545, 475

Stone, J. M., Xu, J., \& Hardee, P. E. 1997, ApJ, 483, 136

Thiele, M., \& Camenzind, M. 2002, A\&A, 381, L53

Tóth, G. 1996, Astrophys. Lett. Commun., 34, 245

Zhao, J. H., Burns, J. O., Norman, M. L., \& Sulkanen, M. E. 1992, ApJ, 387, 83 